\title{
Physics of magnetic resonance imaging: from spin to pixel
}

Gossuin Yves ${ }^{1}$, Hocq Aline ${ }^{1}$, Gillis Pierre ${ }^{1}$ and Vuong Quoc Lam ${ }^{1}$

${ }^{1}$ Biological Physics Department, University of Mons - UMONS, 20 Place du Parc, 7000 Mons, BELGIUM.

\begin{abstract}
:
Magnetic resonance imaging has become a major diagnostic tool in modern medicine. MRI makes it possible to obtain highly contrasted body images without ionizing radiation. Using the magnetic properties of hydrogen protons, MRI is at the crossroads between physics, electronics, signal processing and informatics. In this introductory text, the basic physical principles of MRI image formation are explained. Some important clinical applications are also presented.
\end{abstract}




\section{Introduction}

For thirty years now, Magnetic Resonance Imaging (MRI) has been routinely used in hospitals worldwide as a non-invasive and non-ionizing imaging technique. Based on the nuclear magnetic resonance phenomenon observed in 1946 for protons by Bloch and Purcell, MRI was developed only in 1973 by Lauterbur and Mansfield, notably because it required some important progress in technology and informatics. For their contributions to the development of MRI, Paul Lauterbur and Peter Mansfield received the Nobel Prize in medicine in 2003. The evolution of the initial method has been vertiginous: the time needed to acquire an image has been divided by 100 , the resolution is now below the millimeter, and the development of new imaging sequences is a science in itself. There are numerous MRI applications in the clinical field, including the detection of tumors, functional imaging of the brain, angiography, brain fiber analysis, parallel imaging ... MRI has also found industrial applications, in the food industry for example.

MRI is based on quantum mechanics, uses advanced technology for creating strong magnetic field gradients, for radiofrequency excitation and detection. The image formation is based on mathematical transformations executed by efficient informatics systems. This wide variety of disciplines involved in MRI, combined with the mainly biological applications, makes it sometimes difficult to understand. The aim of this short - and incomplete - introduction to MRI is to give a basic description of the physical principles of this imaging technique as well as some examples of its clinical applications.

\section{Nuclear Magnetic Resonance ${ }^{1,2,3,4,5,6,7}$}

\section{Magnetic properties of the atomic nucleus}

Each nucleus possesses a kinetic momentum J, related to its spin I by:

$$
\vec{J}=\hbar \vec{I}
$$


The measurable values of the projection of the kinetic momentum in a random direction, arbitrarily taken as the $z$-axis, are thus $J_{z}=\hbar I, \hbar(I-1), \hbar(I-2), \ldots,-\hbar(I-2),-\hbar(I-1),-\hbar I$. One can assign a magnetic moment to the nucleus thanks to the relation:

$$
\vec{\mu}=\gamma \vec{J}
$$

where $\gamma$ is the gyromagnetic ratio of the nucleus, $\gamma=g_{n} \beta / \hbar, g_{\mathrm{n}}$ is the Landé factor of the studied nucleus and $\beta=e \hbar / 2 m_{p}$ is the nuclear Bohr magneton. The projection of the nucleus magnetic moment in the z-direction is thus also quantified, $\mu_{z}=\gamma J_{z}$. Only the nuclei having a nonnull magnetic moment - thus a non-null spin - are observable by Nuclear Magnetic Resonance (NMR). The resulting spin of a nucleus depends on the number of protons and neutrons it contains. It is non-null if the number of protons is odd or if it is even with an odd number of neutrons. The NMR behavior may thus be different for isotopes of the same element. The NMR sensitivity of a given isotope takes into account its natural abundance and the amplitude of the NMR signal it produces, depending on its gyromagnetic ratio (table 1 ).

\begin{tabular}{ccccc}
\hline Nucleus & Spin I & $\begin{array}{c}\text { Gyromagnetic ratio } \gamma \\
{\left[\mathbf{1 0}^{7} \mathrm{~T}^{-1} \mathbf{s}^{-1}\right]}\end{array}$ & $\begin{array}{c}\text { Natural abundance } \\
{[\%]}\end{array}$ & $\begin{array}{c}\text { NMR } \\
\text { sensitivity } \\
\text { relative to }{ }^{1} \mathbf{H} \\
1.00\end{array}$ \\
${ }^{1} \mathrm{H}$ & 26.752 & 99.985 & $1.7610^{-4}$ \\
${ }^{13} \mathrm{C}$ & $1 / 2$ & 6.728 & 1.108 & $1.0010^{-3}$ \\
${ }^{14} \mathrm{~N}$ & 1 & 1.934 & 99.63 & $1.0810^{-5}$ \\
${ }^{17} \mathrm{O}$ & $5 / 2$ & -3.628 & 0.037 & 0.83 \\
${ }^{19} \mathrm{~F}$ & $1 / 2$ & 25.181 & 100 & \\
\hline
\end{tabular}

Table 1: NMR sensitivity of different nuclei.

In the rest of this article, only the case of protons (hydrogen nuclei) will be discussed, since MRI uses the NMR signal from the protons of the body, even if some imaging applications use other NMR 
active nuclei. It should be noted that the nuclear magnetic properties of ${ }^{13} \mathrm{C}$ and ${ }^{14} \mathrm{~N}$ are intensively used in NMR spectroscopy, which is another application of nuclear magnetic resonance.

\section{Protons under a magnetic field}

\section{Quantum approach}

For a proton, the possible values of $\mu_{\mathrm{z}}$ are $\mu_{z}= \pm \gamma \hbar / 2$ since $I=1 / 2$. In the absence of a magnetic field, there is degeneracy because the energies corresponding to these two values of $\mu_{2}$ are equal. However, if a magnetic field $\vec{B}_{0}$ is applied along the z-direction, the energy levels will split because of the Zeeman coupling between the nuclear magnetic moment and the magnetic field (figure 1). This can be described by the following Hamiltonian:

$$
H=-\vec{\mu} \cdot \vec{B}_{0}
$$

The two eigenvalues $E_{+}$and $E_{\text {. }}$ of this Hamiltonian correspond to the energies of the protons with $\mu_{\mathrm{z}}$ respectively aligned and anti-aligned with the magnetic field:

$$
E_{+}=-\gamma \hbar B_{0} / 2 \text { and } E_{-}=+\gamma \hbar B_{0} / 2
$$

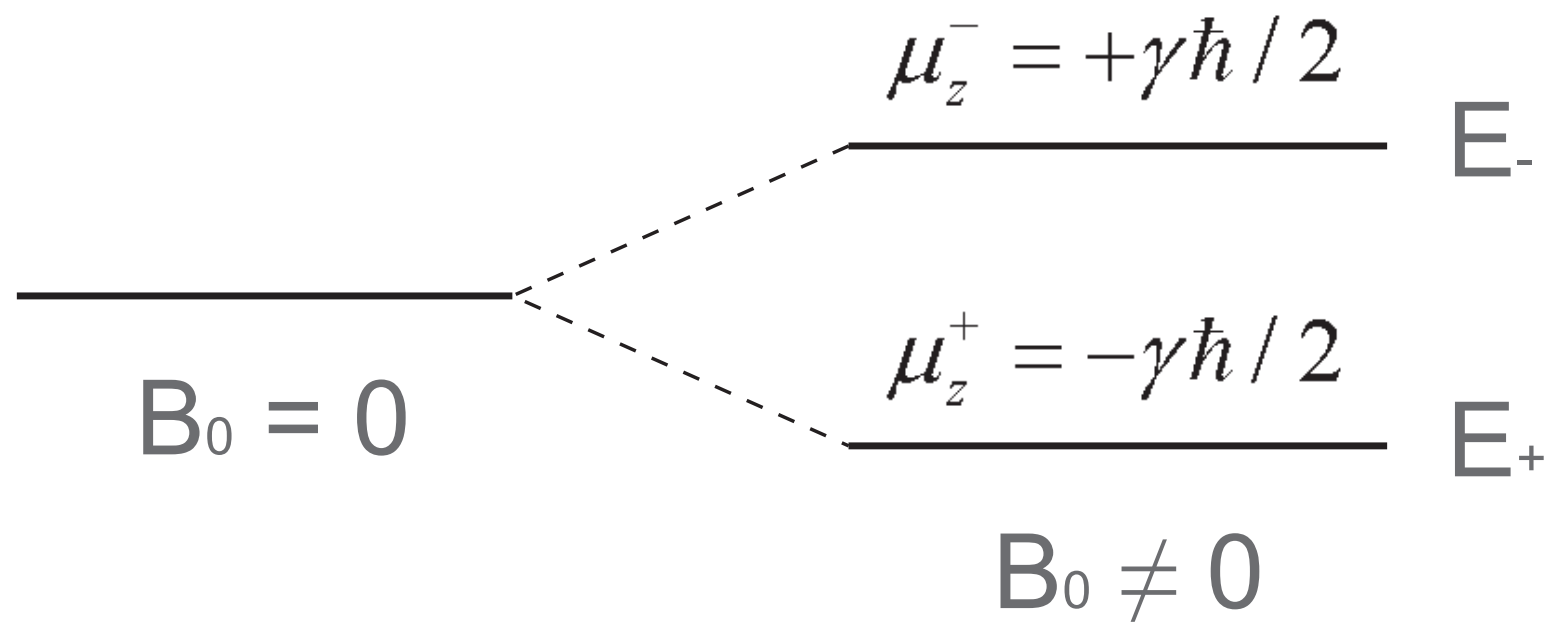

Figure 1: Zeeman splitting of the proton energy levels. 
The energy difference between the two levels is simply given by:

$$
\Delta E=\hbar \gamma B_{0}=\hbar \omega_{0}
$$

Where $\omega_{0}=\gamma B_{0}$ is called the Larmor angular frequency. The excitation of a proton from the $E_{+}$ level to the $E$. level requires a photon with the right energy, $\Delta E$, and therefore the right frequency, called the Larmor frequency:

$$
v_{0}=\gamma B_{0} / 2 \pi
$$

For a population of $\mathrm{N}$ protons at a constant temperature, the distribution of the two energy levels will follow a Boltzmann statistic. If $N_{+}$and $N_{-}$are respectively the number of protons with $E_{+}$ and $E_{\text {., }}$ one obtains:

$$
\frac{N_{+}}{N_{-}}=e^{\frac{\hbar \omega_{0}}{k T}}
$$

$T$ is the absolute temperature and $k$ the Boltzmann constant. For usual magnetic fields, $N_{+} \gtrsim N_{-}$, there are a few more protons with $\mu_{\mathrm{z}}$ aligned than anti-aligned with $\vec{B}_{0}$. The system of protons behaves like a paramagnet: under the application of a magnetic field, it acquires a magnetic moment with the same direction as the field. It can be calculated using a first order development equation (7):

$$
\mu_{0}=N_{+} \mu_{z}^{+}+N_{-} \mu_{z}^{-}=N \frac{(\gamma \hbar)^{2}}{4 k T} B_{0}
$$

For example, one can calculate the magnetic moment of protons of the human body when they are placed inside an MRI scanner using a magnetic field of $3 \mathrm{~T}$. One obtains for a human of $80 \mathrm{~kg}$ whose body contains $70 \%$ water weight, $\mu_{0}=510^{-4} \mathrm{Am}^{2}$. This magnetic moment is of the same order as the "electronic" diamagnetic moment of $20 \mathrm{~g}$ of water in the same magnetic field. This explains why the "static" magnetic properties of nuclei are always overwhelmed by the electronic properties 
of the atoms they belong to. It is thus impossible to directly measure the static equilibrium magnetic moment of a proton population.

\section{Classical approach}

A classical description of the behavior of proton magnetic moments in the presence of a magnetic field can be quite useful for understanding MRI acquisition sequences. In this case, the protons have a classical magnetic moment that is related to their kinetic momentum through:

$$
\vec{\mu}=\frac{e}{2 m} \vec{J}
$$

If we take $\gamma=e / 2 m$, this equation is similar to (2). In a static magnetic field $\vec{B}_{0}$, a torque $\vec{\tau}=\vec{\mu} \times \vec{B}_{0}$ appears that will modify the kinetic momentum according to the classical relation:

$$
\vec{\tau}=\frac{d \vec{J}}{d t}
$$

The evolution of the magnetic moment is thus given by:

$$
\frac{d \vec{\mu}}{d t}=\gamma \vec{\mu} \times \vec{B}_{0}
$$

One can show that (11) describes the precession of the magnetic moment around $\vec{B}_{0}$ at the Larmor frequency $v_{0}=\gamma B_{0} / 2 \pi$. The rotating reference frame $\left(x^{\prime}, y^{\prime}, z\right)$ is defined as the reference frame turning around the $z$-direction at the Larmor frequency, with respectively $\mathrm{x}$ and $\mathrm{x}^{\prime}$, and $\mathrm{y}$ and $\mathrm{y}^{\prime}$ axes coinciding when $t=0$. That frame is especially convenient for describing the dynamics of the magnetic moments. 


\section{Excitation of the system by a radiofrequency field}

To put the system of protons out of equilibrium, we must irradiate the protons with photons having the appropriate frequency $v_{0}=\gamma B_{0} / 2 \pi$. For example, if the static magnetic field is 1 Tesla, the corresponding frequency is $42.6 \mathrm{MHz}$, corresponding to the radiofrequency domain.

From the quantum point of view, this will cause transitions between the Zeeman energy levels, which will perturb their equilibrium populations.

From a classical point of view, we have to use a magnetic field oscillating at $v_{0}$ frequency and perpendicular to $\vec{B}_{0}$, in the x-direction for example. For the rest of the discussion, such a field can be seen as a field $\vec{B}_{1}(t)$, rotating around the z-axis at the Larmor frequency, like the proton magnetic moments ${ }^{1}$. When $\vec{B}_{1}(t)$ is applied, equation (10) becomes:

$$
\frac{d \vec{\mu}}{d t}=\gamma \vec{\mu} \times\left(\vec{B}_{0}+\vec{B}_{1}\right)
$$

In a frame rotating at the pulsation $\omega_{0}$ following the magnetic moments and the rotating field $\vec{B}_{1}(t)$, equation (12) is modified:

$$
\begin{gathered}
\left(\frac{d \vec{\mu}}{d t}\right)_{r o t}=\left(\frac{d \vec{\mu}}{d t}\right)+\omega_{0} \overrightarrow{1}_{Z} \times \vec{\mu}=\gamma \vec{\mu} \times\left(\vec{B}_{0}+\vec{B}_{1}-\frac{\omega_{0}}{\gamma} \overrightarrow{1}_{Z}\right) \\
\vec{B}_{0}=B_{0} \overrightarrow{1}_{Z} \text { and in the rotating frame, } \vec{B}_{1}=B_{1} \overrightarrow{1}_{X^{\prime}} \text {. As } \omega_{0}=\gamma B_{0} \text {, relation (13) simplifies to: } \\
\left(\frac{d \vec{\mu}}{d t}\right)_{r o t}=\gamma \vec{\mu} \times B_{1} \overrightarrow{1}_{X^{\prime}}
\end{gathered}
$$

\footnotetext{
${ }^{1}$ In fact, the magnetic field oscillating at $v_{0}$ can be seen as the sum of two vectors rotating in opposite directions. It can be shown that only the component rotating in the same direction as the precession of the magnetic moment is efficient.
} 
This equation is very similar to relation (11). When an oscillating magnetic field with the Larmor frequency is applied, the resulting magnetic moment of the proton population rotates around the $\vec{B}_{1}$ direction in the rotating frame. $\vec{B}_{1}$ is only efficient if it rotates together with the proton magnetic moment at the Larmor frequency. This is thus a resonance phenomenon. The angular frequency of the rotation of $\vec{\mu}$ around $\vec{B}_{1}$ in the rotating frame is by analogy $\omega_{1}=\gamma B_{1}$. So the flip angle of the magnetic moment is given by:

$$
\alpha=\omega_{1} t=\gamma B_{1} t
$$

By varying the duration $t$ of application of the $B_{1}$ field (or in some cases by varying its amplitude), one can choose to rotate the resulting magnetic moment of the protons with different angles. The most used flip angles are $90^{\circ}$ and $180^{\circ}$, one speaks then of $90^{\circ}$ and $180^{\circ}$ pulses, respectively (figure 2).

After a radiofrequency pulse tuned at the Larmor frequency, the proton system is out of equilibrium - it will return to equilibrium through transitions between the Zeeman energy levels. This could not be achieved by spontaneous transitions: their probability is negligible. In fact the return to equilibrium is caused by time-modulated interactions of the protons with their environment, in a process called relaxation. 

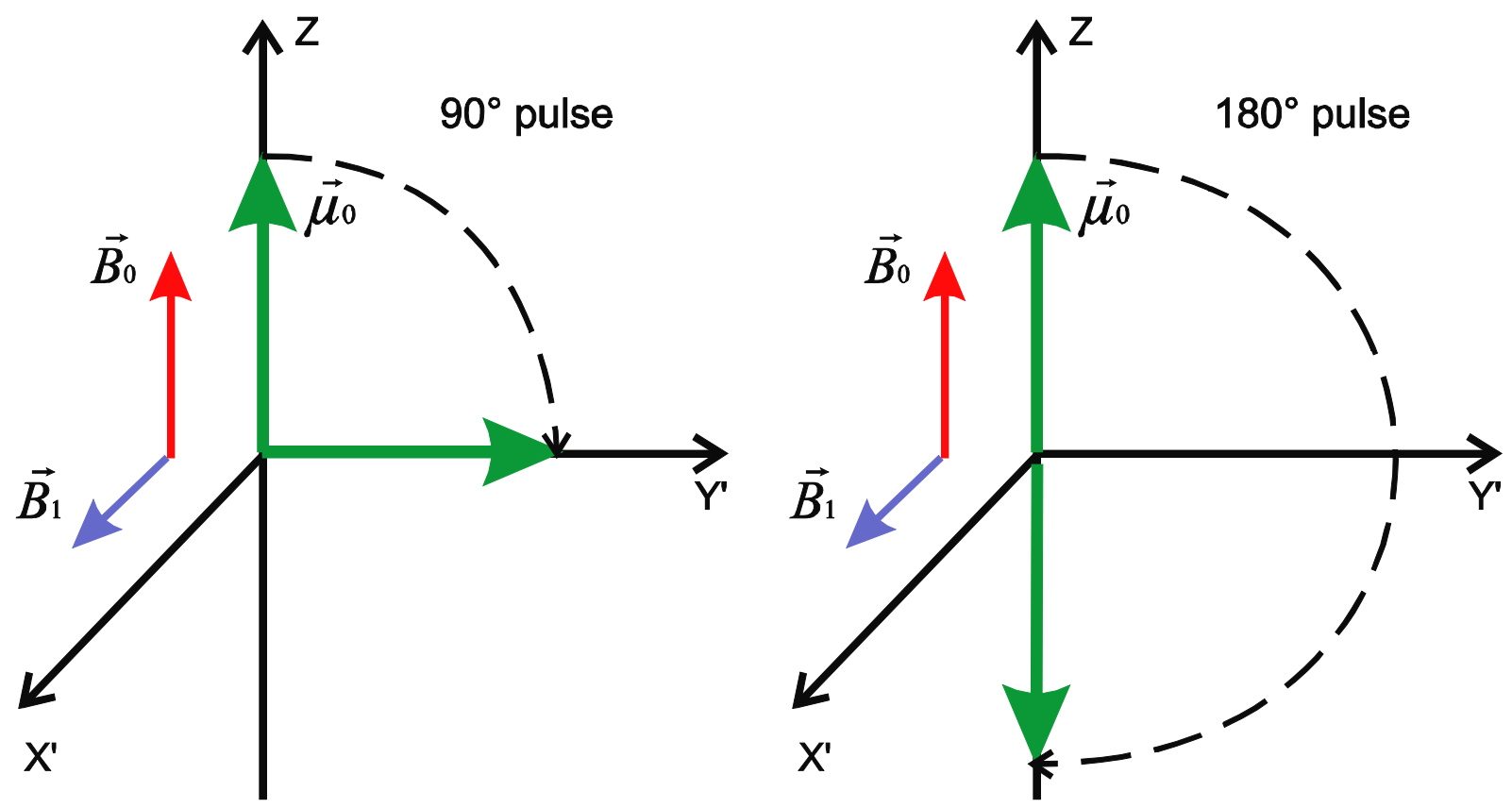

Figure 2: Illustration of $90^{\circ}$ and $180^{\circ}$ radiofrequency pulses in the rotating frame.

\section{NMR relaxation}

After a $180^{\circ}$ pulse, the equilibrium magnetic moment of the proton system is inverted along the z-direction. The z-component of $\vec{\mu}_{0}$, noted $\mu_{z}$, must go from $-\mu_{0}$ just after the pulse to $+\mu_{0}$ at equilibrium. Bloch phenomenologically described the return to equilibrium of $\mu_{z}$ with the following equation:

$$
\frac{d \mu_{Z}(t)}{d t}=-\frac{\left[\mu_{Z}(t)-\mu_{0}\right]}{T_{1}}
$$

This leads to an exponential evolution of $\mu_{z}$ :

$$
\mu_{Z}(t)=\mu_{0}\left[1-2 e^{-t / T_{1}}\right]
$$

The return to equilibrium of $\mu_{z}$ is called the longitudinal relaxation, because it concerns the zcomponent of the magnetic moment. The time constant $T_{1}$ is the longitudinal relaxation time (figure 3). 

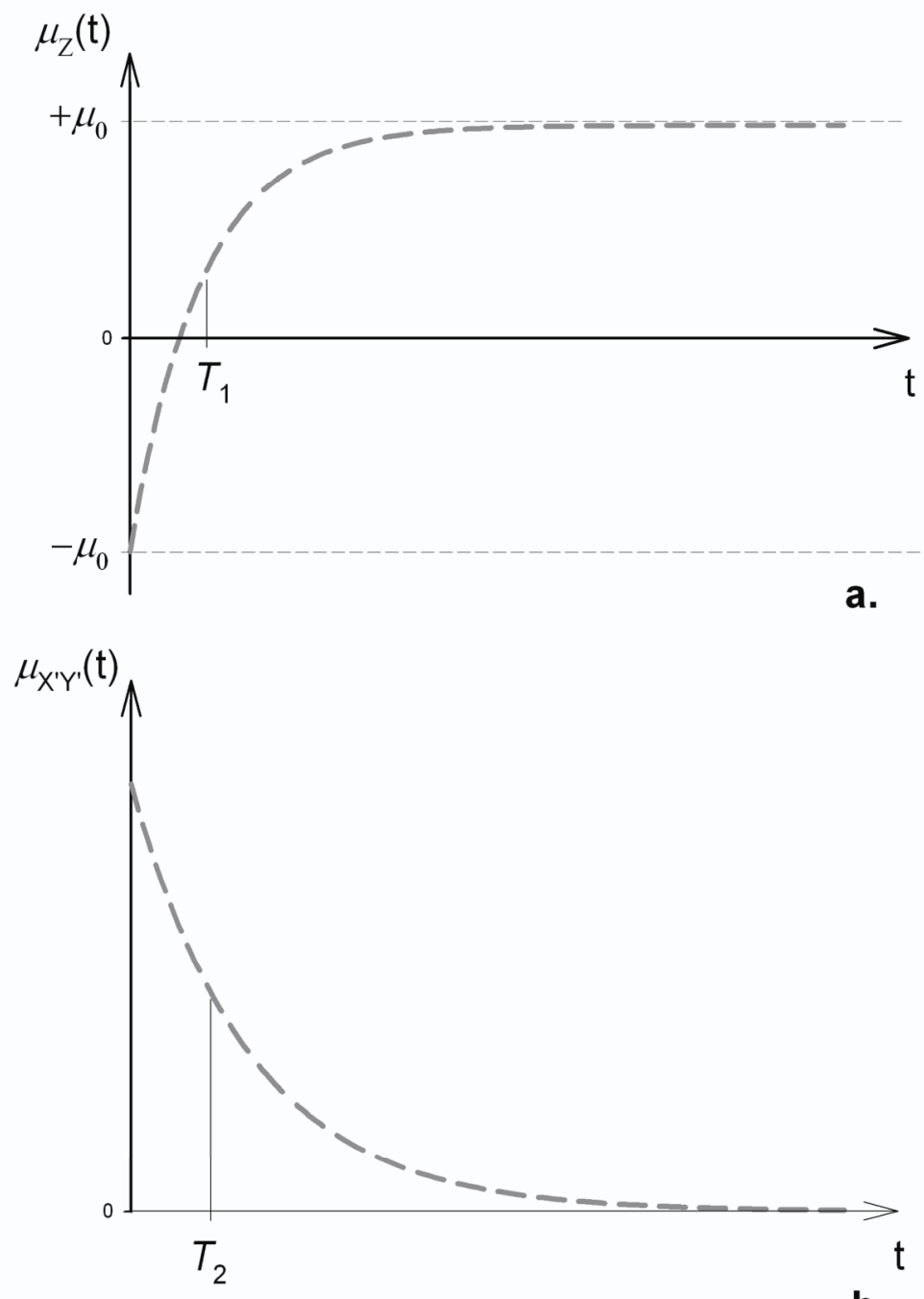

b.

Figure 3: Illustration of the return to equilibrium of (a) $\mu_{z}$ after a $180^{\circ}$ radiofrequency pulse and (b) $\mu_{X^{\prime} Y^{\prime}}$ after a $90^{\circ}$ pulse.

On the other hand, after a $90^{\circ}$ pulse, the magnetic moment is in the transverse plane $X^{\prime} Y^{\prime}-$ i.e. the plane perpendicular to the $B_{0}$ field direction. Let us call $\mu_{X^{\prime} Y^{\prime}}$ the transverse component of the magnetic moment in the rotating frame; it is null at equilibrium, $\mu_{X^{\prime} Y^{\prime}}$ must thus cancel during relaxation. The return to equilibrium of $\mu_{X^{\prime} Y^{\prime}}$, is called the transverse relaxation, because it concerns 
the transverse component of the magnetic moment. Transverse relaxation can be described by the following Bloch equations:

$$
\begin{gathered}
\frac{d \mu_{X^{\prime} Y^{\prime}}(t)}{d t}=-\frac{\mu_{X^{\prime} Y^{\prime}}(t)}{T_{2}} \\
\mu_{X^{\prime} Y^{\prime}}(t)=\mu_{0} e^{-t / T_{2}}
\end{gathered}
$$

The time constant $T_{2}$ is called the transverse relaxation time. After a rotation by an arbitrary angle, the return to equilibrium of the magnetic moment is given by the solution of the system of equations (16) and (18). $T_{1}$ and $T_{2}$ are not necessarily equal ( $\left.T_{1} \quad T_{2}\right)$ since some processes participate in transverse relaxation and not in longitudinal relaxation. The relaxation times are dependent on the magnetic field $B_{0}$ and temperature. For pure water, the $T_{1}$ and $T_{2}$ of the proton are $\sim 4 \mathrm{~s}$, but in most biological tissues $T_{2} \ll T_{1}$. Knowing the relaxation times is crucial for MRI, since the relaxation governs the signal intensity and therefore the image contrast.

\section{NMR signal and basic experimental setup}

We have already seen that a static nuclear magnetic moment is difficult to detect. However, after a $90^{\circ}$ pulse, the magnetic moment is perpendicular to $\vec{B}_{0}$, so that it precesses around the z-axis at the Larmor frequency. This precession causes magnetic flux variations that can be detected by a coil tuned to the Larmor frequency. According to Faraday's law, an induced voltage will appear in the coil. The signal will be proportional to the magnetic moment, which is very low, but also to the flux variation speed, which is given by the Larmor frequency. As this latter is of the order of tens of $10^{6} \mathrm{~Hz}$ for usual magnetic fields, the induced signal is measurable.

As a consequence, in NMR and MRI, only the transverse component of the nuclear magnetic moment can be detected. A $90^{\circ}$ pulse is thus used before any measurement of the magnetic moment. Figure 4 shows the basic NMR experimental setup. 


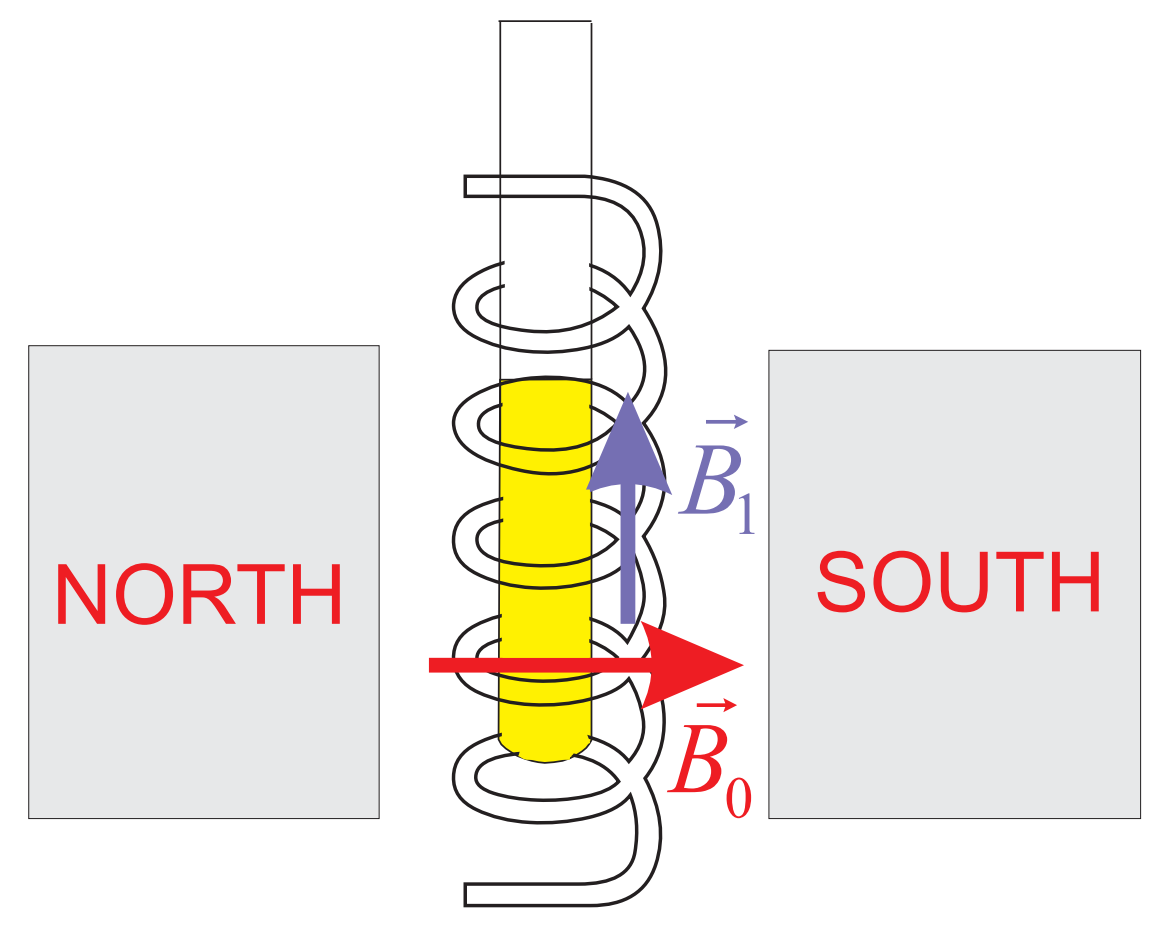

Figure 4: Basic NMR experimental setup, with the permanent magnet and the excitation-detection coil.

After a $90^{\circ}$ pulse, the detection coil will thus measure an electric signal oscillating at the Larmor frequency. This oscillation is removed by the detection electronics, and the resulting signal is simply an exponential decay, called the Free Induction Decay (FID). Ideally, the time constant of this decay should be $T_{2}$, but the signal decays more rapidly, in fact, with a $T_{2}{ }^{*}$ time constant. This fast decay is due to the dephasing of the proton magnetic moments in the transverse plane just after the $90^{\circ}$ pulse. This dephasing is explained by the spatial inhomogeneities of the $\vec{B}_{0}$ field, which causes variations of the precession frequency among magnetic moments situated at different positions of the sample: some magnetic moments rotate faster than the mean; some others rotate more slowly than the mean. This dephasing phenomenon is illustrated in the rotating frame in figure 5 . It causes a loss of signal whose time constant is $T_{2}{ }^{*}$. 


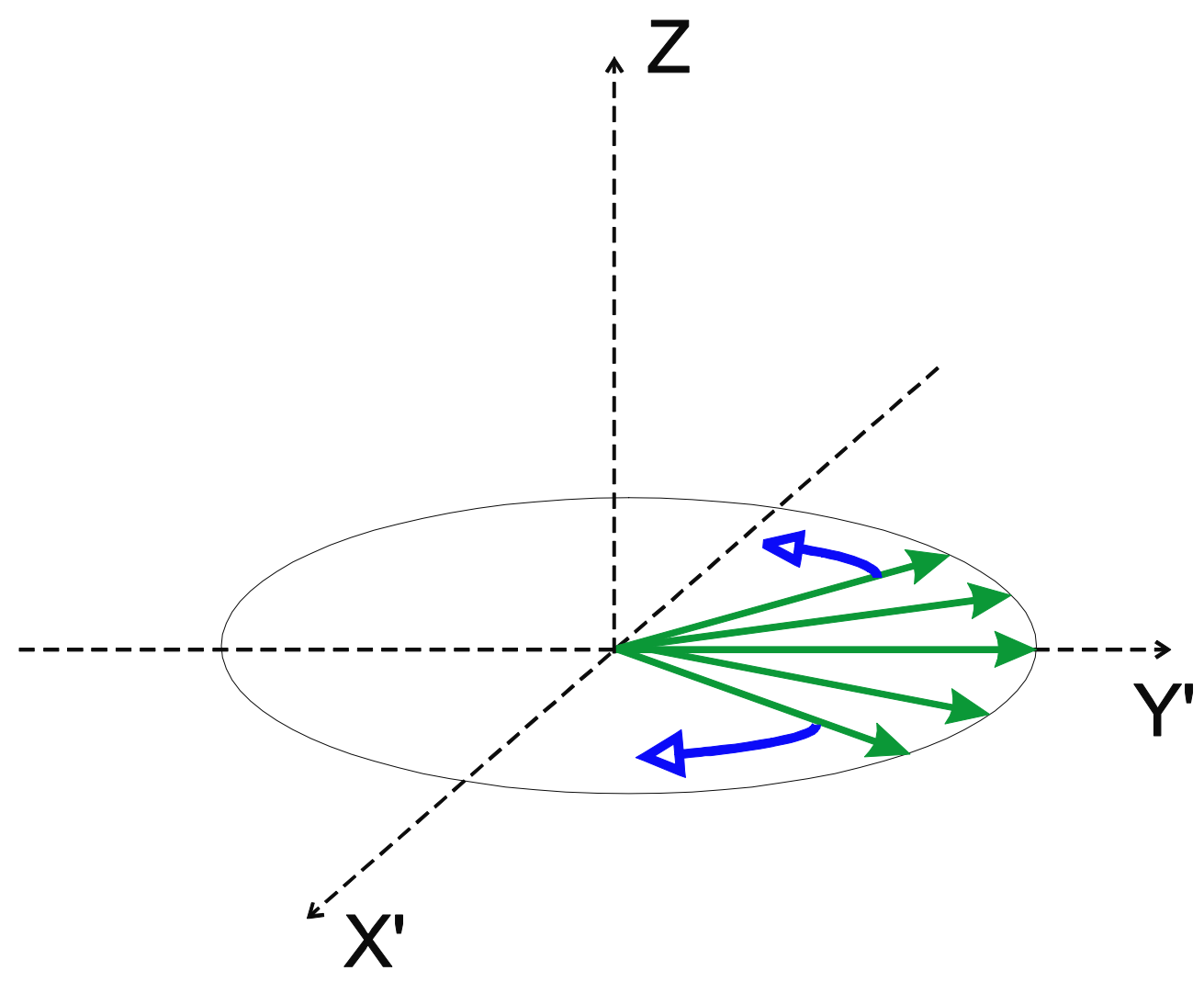

Figure 5: Dephasing of the proton magnetic moments in the rotating frame after a $90^{\circ}$ pulse on the $1_{X^{\prime}}$ direction.

\section{Measurement of $T_{1}$ and $T_{2}$}

Except in some specific applications, MRI is not used to measure relaxation times. However, the sequences used in conventional NMR for the measurement of $T_{1}$ and $T_{2}$ are useful to introduce the basic MRI sequences and contrast mechanisms.

\section{Inversion-recovery for $T_{1}$}

The most popular sequence for the measurement of $T_{1}$ is the inversion-recovery, illustrated in figure 6 . The magnetic moment is first inverted by a $180^{\circ}$ pulse. After a relaxation delay $\tau$, while $\mu_{z}$ partially returns to equilibrium, $\mu_{z}$ is rotated in the transverse plane by a $90^{\circ}$ pulse, and its amplitude is measured. After this measurement, a repetition delay of at least $5 T_{1}$ is respected, in order to get back to equilibrium, and a new sequence is started for a different value of $\tau$. This is repeated $\mathrm{n}$ times 
for $\mathrm{n}$ different values of $\tau$, and an inversion-recovery curve with $\mathrm{n}$ points is constructed (figure $3 a) . T_{1}$ is obtained when equation (17) is fitted to the data.
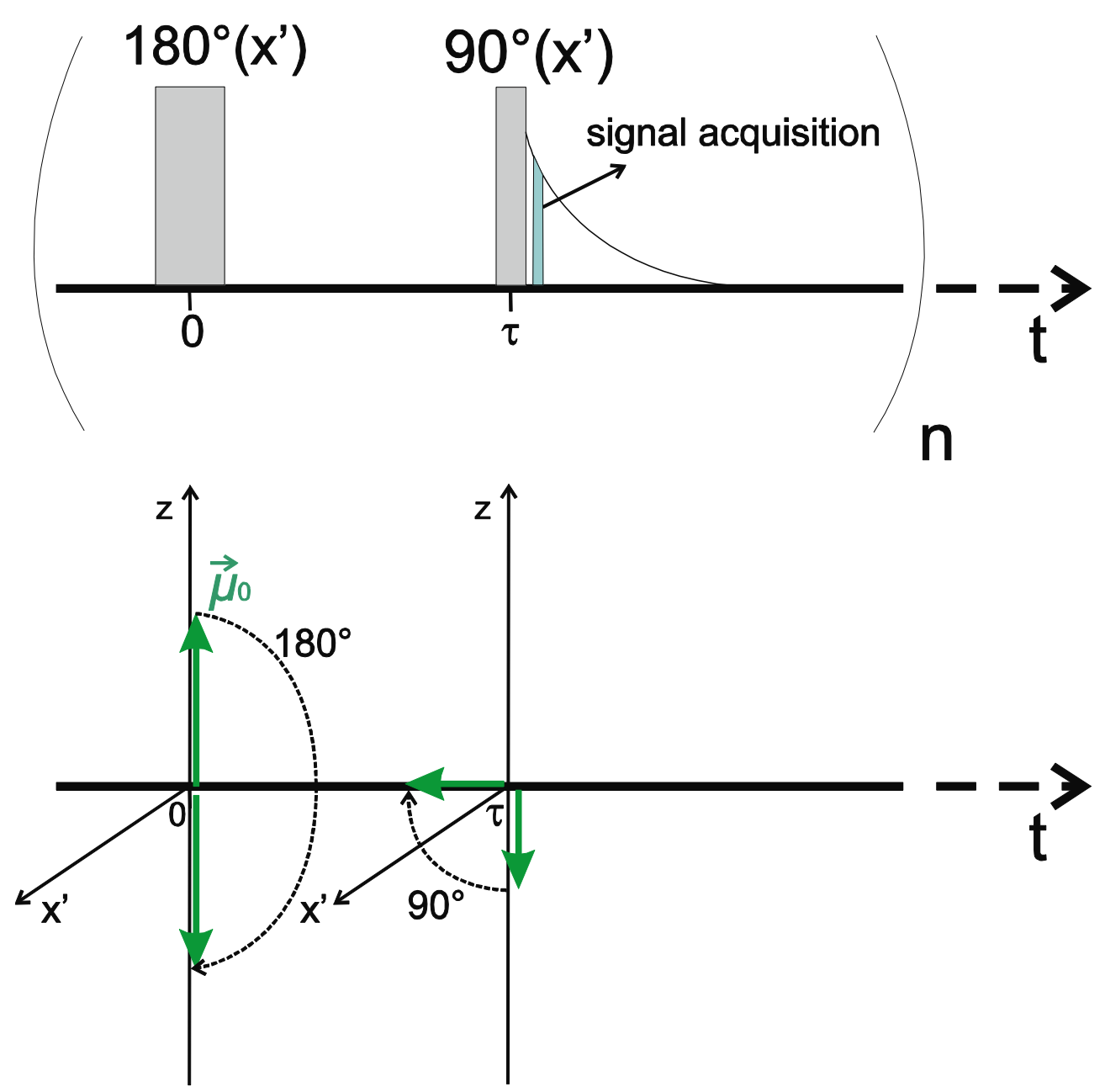

Figure 6: Inversion-recovery sequence for the measurement of $T_{1}$ ( (a) diagram of the pulses, (b) evolution of the proton magnetic moment.

\section{Spin echo for $T_{2}$}

As seen above, after a $90^{\circ}$ pulse, the magnetic moments defocus in the transverse plane, because of spatial variations of precession speed within the sample. This process is reversible and can be cancelled by using a $180^{\circ}$ pulse applied at $\mathrm{t}=\mathrm{TE} / 2$ on the $\mathrm{y}^{\prime}$ axis. Then the most rapid magnetic moments are delayed and the slower ones are given an advance. This leads to a refocusing of the magnetic moments at $\mathrm{t}=\mathrm{TE}$, corresponding to a maximum signal called the spin echo. This 
process is illustrated in figure 7. For the sake of clarity, only the magnetic moments rotating faster than the mean are illustrated. To measure $T_{2}$, this sequence should be repeated (with a $5 T_{1}$ repetition time) for $n$ different values of TE. The evolution curve of the transverse magnetization can be reconstructed and a fitting of equation (19) to the data provides $T_{2}$.
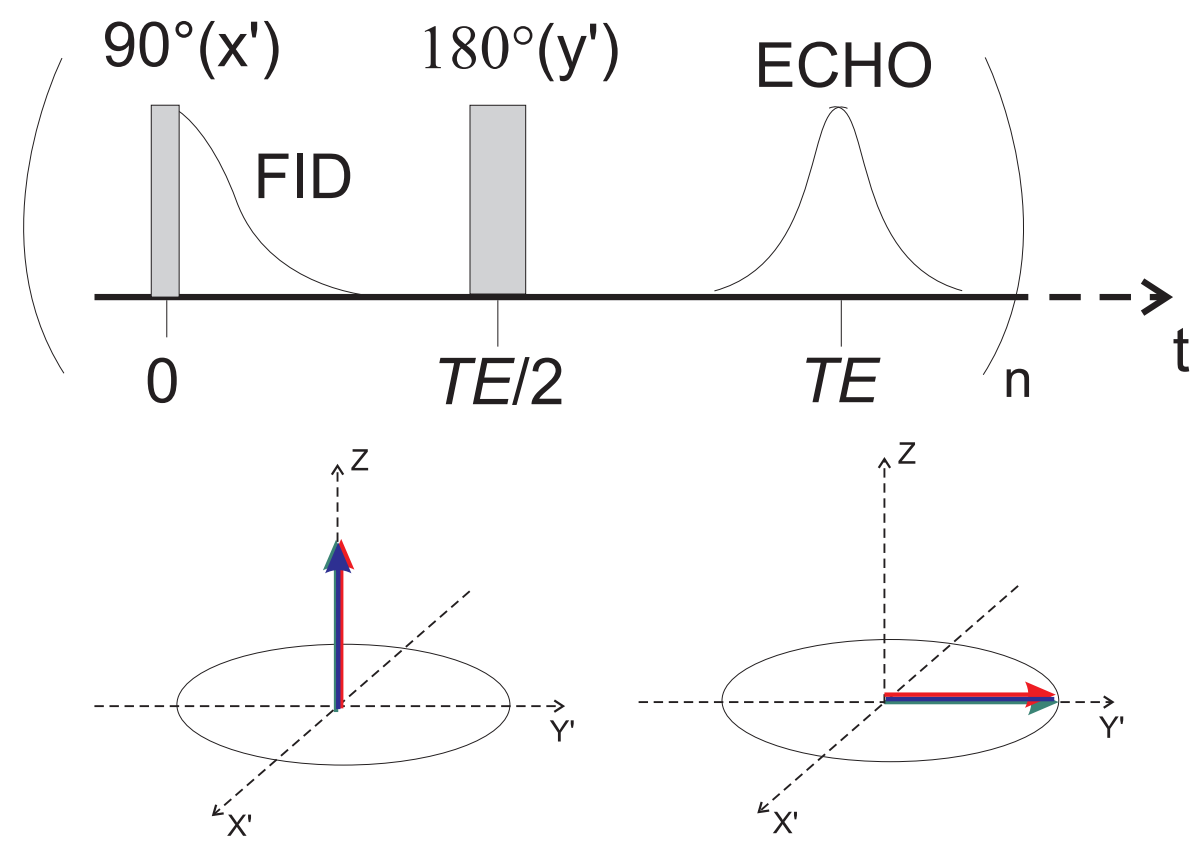

magnetic moments at equilibrium

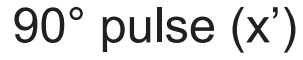

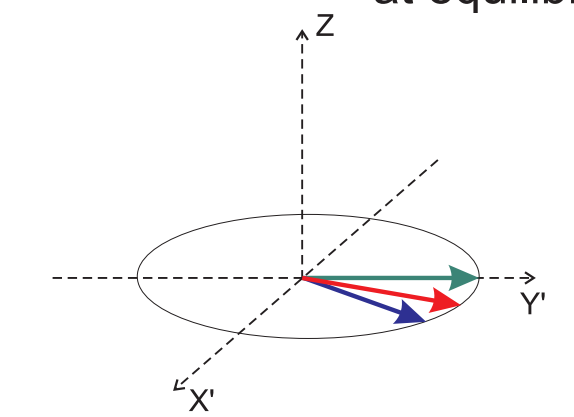

defocusing of the magnetic moments after $\mathrm{t}=T E / 2$

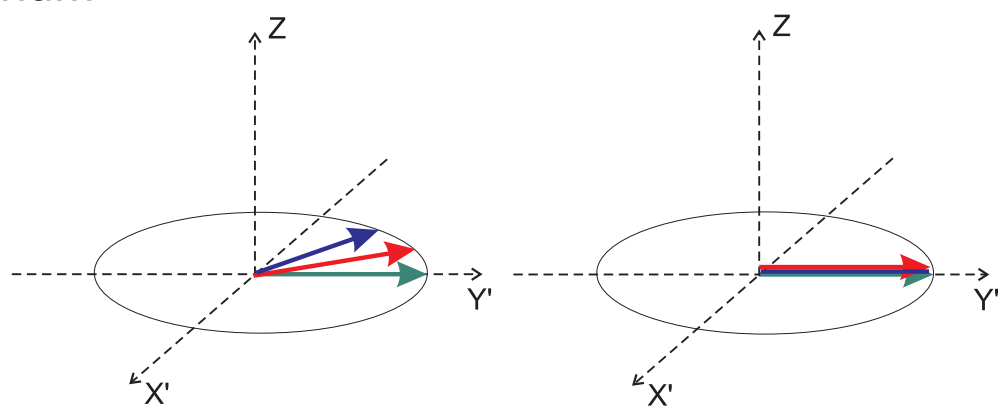

$180^{\circ}$ pulse $\left(y^{\prime}\right)$ refocusing after $t=T E$ $=>\mathrm{ECHO}$

Figure 7: (a) Spin echo sequence for the measurement of $T_{2}$, (b) evolution of the transverse magnetic moments during this sequence.

Because of the mobility of the protons within the sample, the $180^{\circ}$ pulse is only efficient for short echo times for which the precession speeds of the protons are the same before and after the 
refocusing pulses. Another sequence was thus developed to avoid using long echo time, the CarrPurcell-Meiboom-Gill (CPMG) sequence. This technique (figure 8) uses regularly-spaced successive $180^{\circ}$ pulses and successive echoes are thus obtained in one single measurement, allowing a significant gain of time.

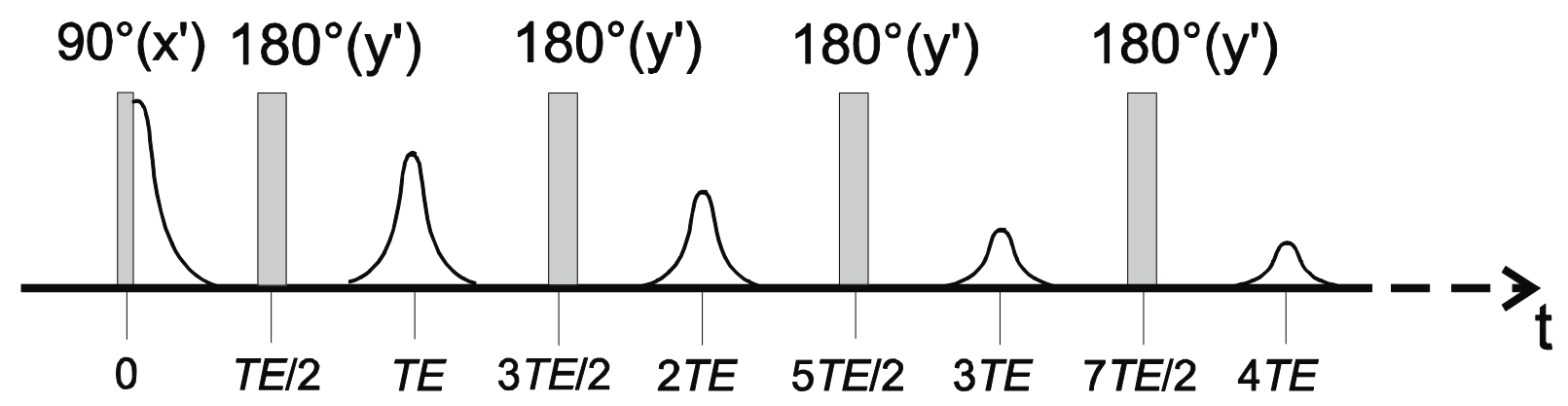

Figure 8: CPMG sequence for the measurement of $T_{2}$.

\section{Magnetic Resonance Imaging 8,9,10,11,12}

The human body is composed of $70 \%$ water weight. The water molecule bears two hydrogen protons and several other molecules of our body, like fat, also bear protons. Our body is therefore an ideal object for a proton NMR experiment. However, the signal obtained with these in vivo protons is at first glance useless to obtain an image. The brilliant idea from Paul Lauterbur was to use magnetic field gradients ${ }^{2}$ to "tag" the signal from protons according to their spatial position.

\section{Slice selection}

The first step is to select a 2-dimensional slice of the body. If, as usual, $\mathrm{z}$ is the direction of the static magnetic field, this slice selection is achieved thanks to a magnetic field gradient $G_{\mathrm{z}}$ along the zdirection:

$$
B_{0}=B_{0 m}+G_{z} \cdot z
$$

\footnotetext{
${ }^{2}$ The magnitude of the gradients used in MRI is comprised between 0.01 and $0.1 \mathrm{~T} / \mathrm{m}$. For MRI research on small animals, the gradients can reach $0.6 \mathrm{~T} / \mathrm{m}$.
} 
The magnetic field thus increases linearly with the z-coordinate. As a consequence, the Larmor frequency (given by equation 6) also depends linearly on z (figure 9). It is thus possible to selectively excite protons belonging to a slice perpendicular to the z-axis by choosing an excitation pulse $\left(a 90^{\circ}\right.$ pulse, for example) having the exact frequency corresponding to the Larmor frequency of the slice protons. To change the position of the slice upwards or downwards, the excitation pulse frequency simply has to be increased or decreased.

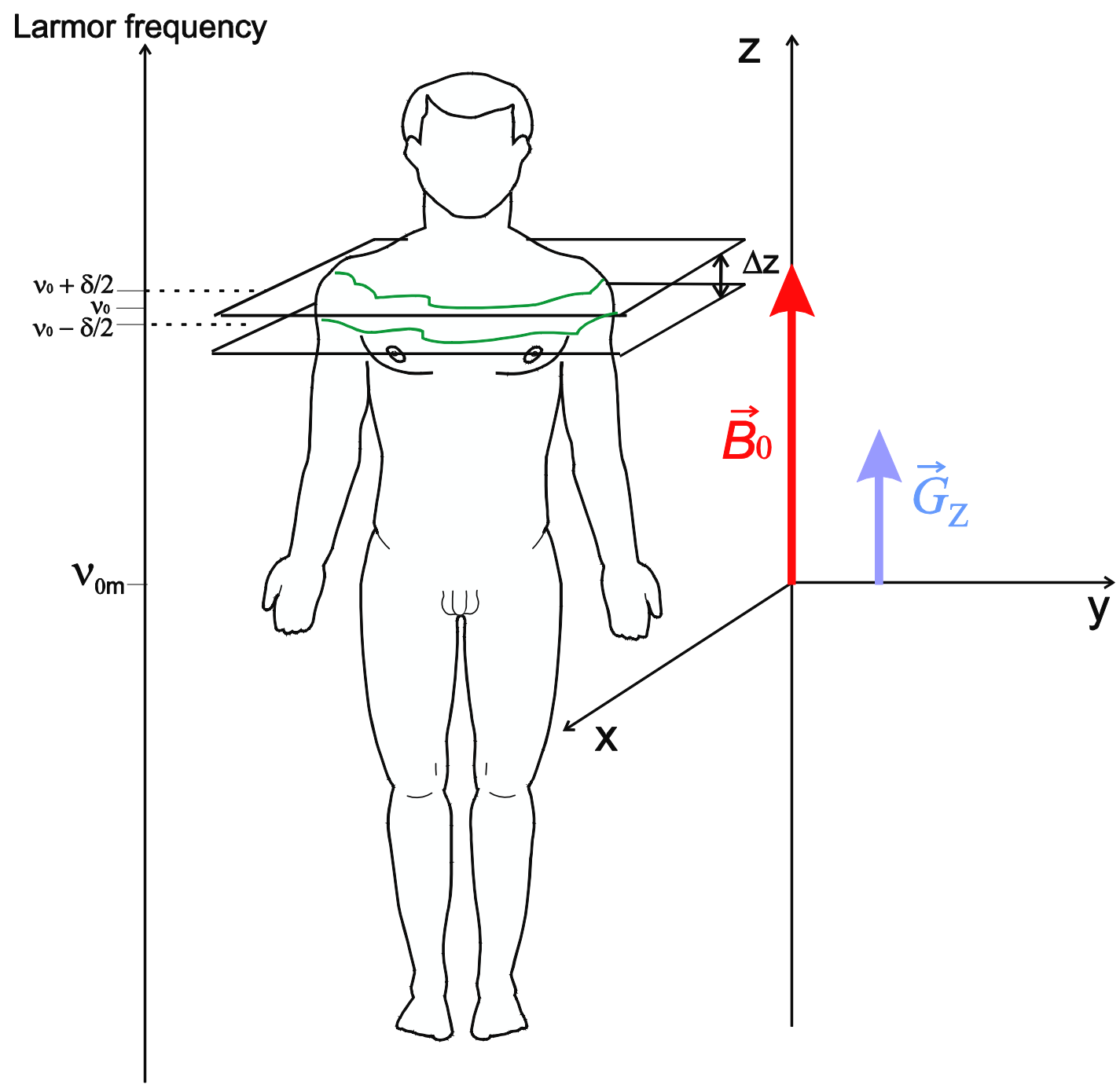

Figure 9: Principle of slice selection.

The spatial width $\Delta z$ of the slice depends on the frequency width of the excitation. The frequency distribution of the radiofrequency pulse is obtained thanks to the Fourier Transform (FT) of the time profile of the pulse (figure 10). The spatial excitation profile in the slice-select direction is thus also 
approximately given by the FT of the pulse. A rectangular pulse in the time domain will thus produce a sinc profile centered at $v_{0}$ in the frequency domain. The definition of the slice will not be optimal in this case because of the presence of non-negligible lateral lobes of the sinc function. As a consequence, more elaborate pulse envelopes in the time domain have to be used: for example an infinite sinc pulse profile in the time domain provides a perfect rectangular profile in the frequency domain, and thus a perfect rectangular slice. Real pulses are of course truncated in time, which affects the spatial shape of the slice. Other profiles (Gaussian, hyperbolic...) are often used for radiofrequency pulses in MRI sequences.
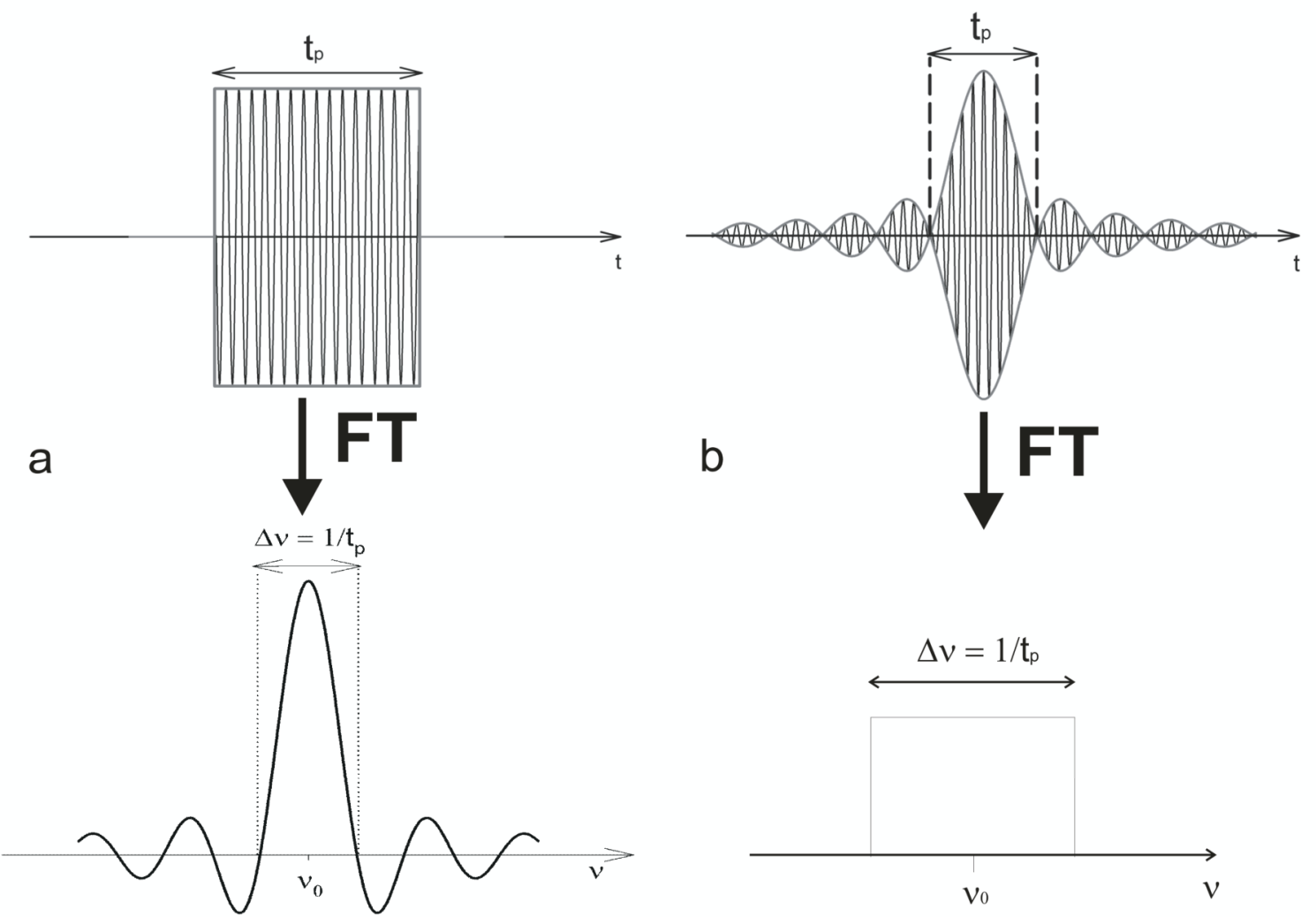

Figure 10: Slice selection pulse profile in the time domain and in the frequency domain; (a) rectangular pulse and (b) sinc pulse.

Slice selection is an important step in the construction of an image by Magnetic Resonance, but in the selected slice, how is it possible to distinguish the protons according to their $(x, y)$ coordinate? Gradients along the $\mathrm{x}$ - and $\mathrm{y}$-directions will be used at the right moment of the measuring sequence 
in order to achieve the so-called frequency encoding and phase encoding of the signal. For the following two paragraphs, let us consider that a slice-selective $90^{\circ}$ pulse has been made, and that the frequency and phase encoding of the precession signal has to be performed.

\section{Frequency encoding}

If we apply a gradient along the $x$-direction ${ }^{3}$, the precession frequency of the protons will depend on their position on the $x$-axis (figure 11). This will be used to distinguish protons with different $x$ coordinates. The frequency encoding is performed by applying the $G_{x}$ gradient during the acquisition of the signal.

\footnotetext{
${ }^{3}$ It should be stressed that a gradient on the $x$-direction does not change the direction of the magnetic field, which is still aligned in the $z$-direction. It simply means that the protons will have different $B_{0}$ amplitudes depending on their position on the $x$-axis.
} 


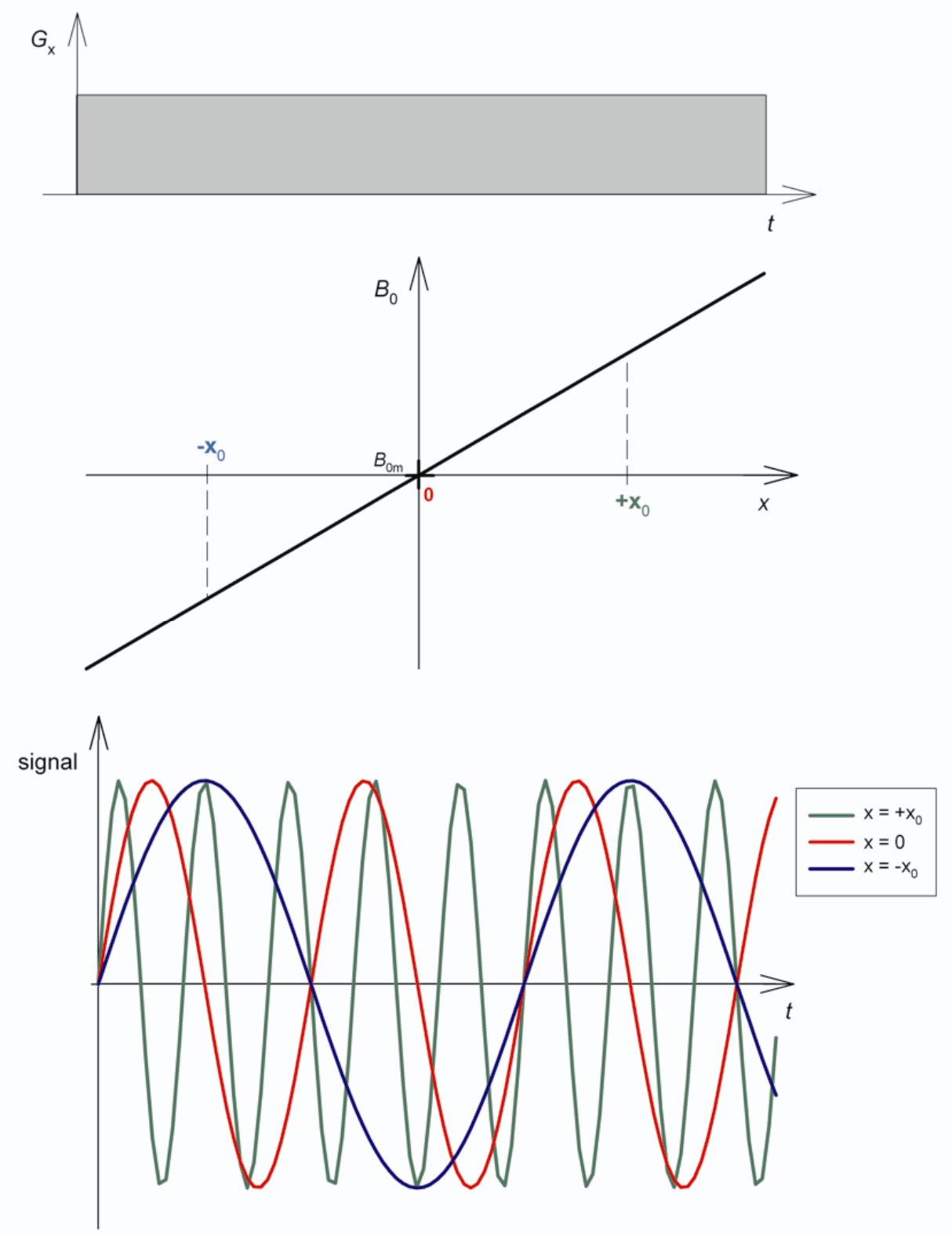

Figure 11: Principle of frequency encoding.

\section{Phase encoding}

As the acquisition of the signal is used to "detect" the $\mathrm{x}$ coordinate thanks to frequency encoding, one must find another way to tag the signal according to the y coordinate of the protons. This is done thanks to the application of a gradient pulse along the y direction before the acquisition of the signal. Because of this gradient, the signal of protons situated at different y positions will present different phases. 

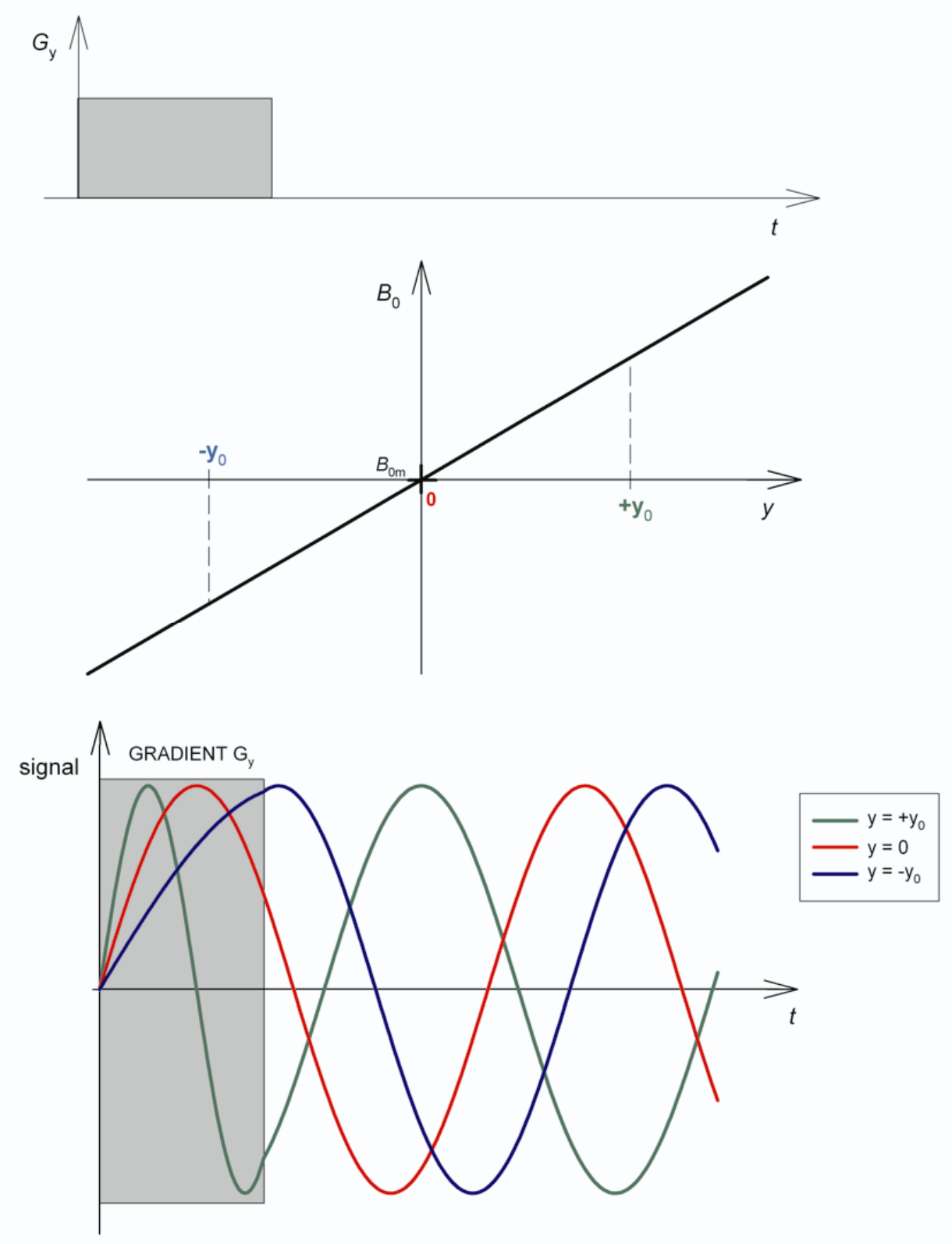

Figure 12: Principle of phase encoding

\section{Imaging and $k$-space ${ }^{8,9,12,13}$}

After the slice selection, it is now intuitively clear how the frequency encoding and the phase encoding gradients make it possible to mark the signal according to the $(x, y)$ coordinate of the protons. It is, however, much less obvious to understand how an "image of the protons" can be obtained from their time-dependent MR signal. After acquisition, the NMR signal is amplified and demodulated: the oscillation at the Larmor frequency of the detected voltage is cancelled. If we 
neglect the effects of relaxation, the demodulated signal obtained during the frequency encoding is simply given by:

$$
\begin{aligned}
& d S(t, x, y)=I(x, y) e^{-i \gamma G_{x} x t} d x d y \\
& \text { with } I(x, y)=\int_{-\Delta z / 2}^{+\Delta z / 2} \rho(x, y, z) d z \\
& S(t)=\iint_{\text {slide }} I(x, y) e^{-i \gamma G_{x} x t} d x d y
\end{aligned}
$$

$\rho(x, y, z)$ is the proton density at the $(x, y, z)$ position and $e^{-i \gamma G_{x} x t}$ expresses - in complex notation $^{4}$ - the frequency encoding, which induces different frequencies for signals from protons at different $\mathrm{x}$ positions. The demodulated signal obtained after a phase-encoding gradient pulse of $T_{\mathrm{P}}$ duration is:

$$
\begin{aligned}
& \left\{\begin{array}{l}
d S(t, x, y)=I(x, y) e^{-i \gamma G_{y} y t} d x d y \quad 0<t<T_{P} \\
d S(t, x, y)=I(x, y) e^{-i \gamma G_{y} y T_{P}} d x d y \quad t>T_{P}
\end{array}\right. \\
& S(t)=\iint_{\text {slide }} I(x, y) e^{-i \gamma G_{y} y T_{P}} d x d y \quad t>T_{P}
\end{aligned}
$$

Where $\gamma G_{y} y T_{P}$ is the phase accumulated during the phase-encoding process by the protons with a y coordinate.

The simplest imaging sequence comprising a phase-encoding and a frequency-encoding step is the gradient echo sequence described in figure 14a, representing the timeline of the different radiofrequencies and $G_{z}, G_{x}$ and $G_{y}$ pulses. The $G_{z}$ gradient and the radiofrequency pulses are always applied simultaneously to ensure slice selection. The negative $G_{z}$ gradient following the $90^{\circ}$ pulse is necessary to destroy the phases accumulated by the protons because of the slice selection gradient. Without this compensation, the phase encoding along the y-direction would be perturbed. Such compensation gradients should be used after each excitation pulse.

\footnotetext{
${ }^{4}$ Complex formalism is often used in NMR. The real part of the complex number represents the $x^{\prime}$ component of the nuclear magnetic moment while the imaginary part accounts for its $y^{\prime}$ component.
} 
For the gradient echo sequence the acquired signal is thus given by:

$$
S(t)=\iint_{\text {slide }} I(x, y) e^{-i 2 \pi\left[\not G_{y} T_{P} y+\gamma G_{x}(t-T E) x\right]} d x d y
$$

Phase encoding Frequency encoding

Where $\psi=\gamma / 2 \pi$. If we define new variables, relation (23) can be rewritten:

$$
\begin{aligned}
& k_{x}=\mathcal{H} G_{x}(t-T E) \text { and } k_{y}=\mathcal{H} G_{y} T_{P} \\
& S\left(k_{x}, k_{y}\right)=\iint_{\text {slide }} I(x, y) e^{-i 2 \pi\left[k_{x} x+k_{y} y\right]} d x d y
\end{aligned}
$$

This relation shows that the time-dependent signal is the 2-dimensional Fourier Transform of the spatial distribution of signal $I(x, y)$ in the slice.

$$
S\left(k_{x}, k_{y}\right)=F T[I(x, y)] \text { and } I(x, y)=F T^{-1}\left[S\left(k_{x}, k_{y}\right)\right]
$$

Sampling the $S\left(k_{x}, k_{y}\right)$ function makes it possible to "map" the proton signal in the slice by a mathematical transformation, the Fourier Transform (fig. 13). One must thus record the signal in the so-called " $k$-space". For the gradient echo sequence, a line of $k$-space (along the $k_{\mathrm{x}}$ direction) is sampled during the frequency-encoded signal acquisition. From a practical point of view, the acquired time-dependent signal is digitized for successive $\Delta t$ steps. By definition the number of steps is $N=T_{\mathrm{A}} / \Delta t$, where $T_{\mathrm{A}}$ is the acquisition time. A new line is then recorded by repeating the sequence for a new $k_{y}$ value, obtained by changing the phase-encoding gradient $G_{y}$ with a $\Delta G_{y}$ increment (figure 14a). The total $k$-space is sampled by repeating the sequence for $N$ different values of $G_{y}$ :

$$
\begin{aligned}
& G_{y}^{n}=n \Delta G_{y} \text { with } n=-\frac{N}{2},-\frac{N}{2}+1, \ldots, \frac{N}{2}-1, \frac{N}{2} \\
& G_{y M A X}=\frac{N \Delta G_{y}}{2}
\end{aligned}
$$


Finally an $N \times N$ matrix of $k$-space is obtained, whose 2-dimensional Fourier Transform provides an $N X N$ matrix of $/(\mathrm{x}, \mathrm{y})$, as illustrated in figure 13 . The increments in the $k_{\mathrm{x}}$ and $k_{\mathrm{y}}$ directions are simply dependent on the sequence parameters $\Delta t$ and $\Delta G_{y}$ :

$$
\begin{aligned}
& \Delta k_{x}=\psi\left|G_{x}\right| \Delta t \\
& \Delta k_{y}=\psi \Delta G_{y} T_{P}
\end{aligned}
$$

The field of view (FOV) is defined as the spatial dimension of the image in the $\mathrm{x}$ - and $\mathrm{y}$-directions. In our case, it is simply given by FOV $=2 x_{\mathrm{MAx}}$ and $F O V_{\mathrm{y}}=2 y_{\mathrm{MAx}}$. The resolutions along the $\mathrm{x}$ - and $\mathrm{y}$-axis are given by $\Delta x$ and $\Delta y$. From the Fourier Transform properties and the sampling theory (ref), it follows that:

$$
\begin{aligned}
& F O V_{x}=\frac{1}{\Delta k_{x}}=\frac{1}{\psi G_{x} \Delta t} ; F O V_{y}=\frac{1}{\Delta k_{y}}=\frac{1}{\psi \Delta \Delta G_{y} T_{P}} \\
& \Delta x=\frac{F O V_{x}}{N}=\frac{1}{\not G_{x} T_{A}} ; \Delta y=\frac{F O V_{y}}{N}=\frac{1}{2 \nvdash G_{y M A X} T_{P}}
\end{aligned}
$$

From these equations, it is possible to determine the experimental parameters needed to obtain an image with the desired resolution.

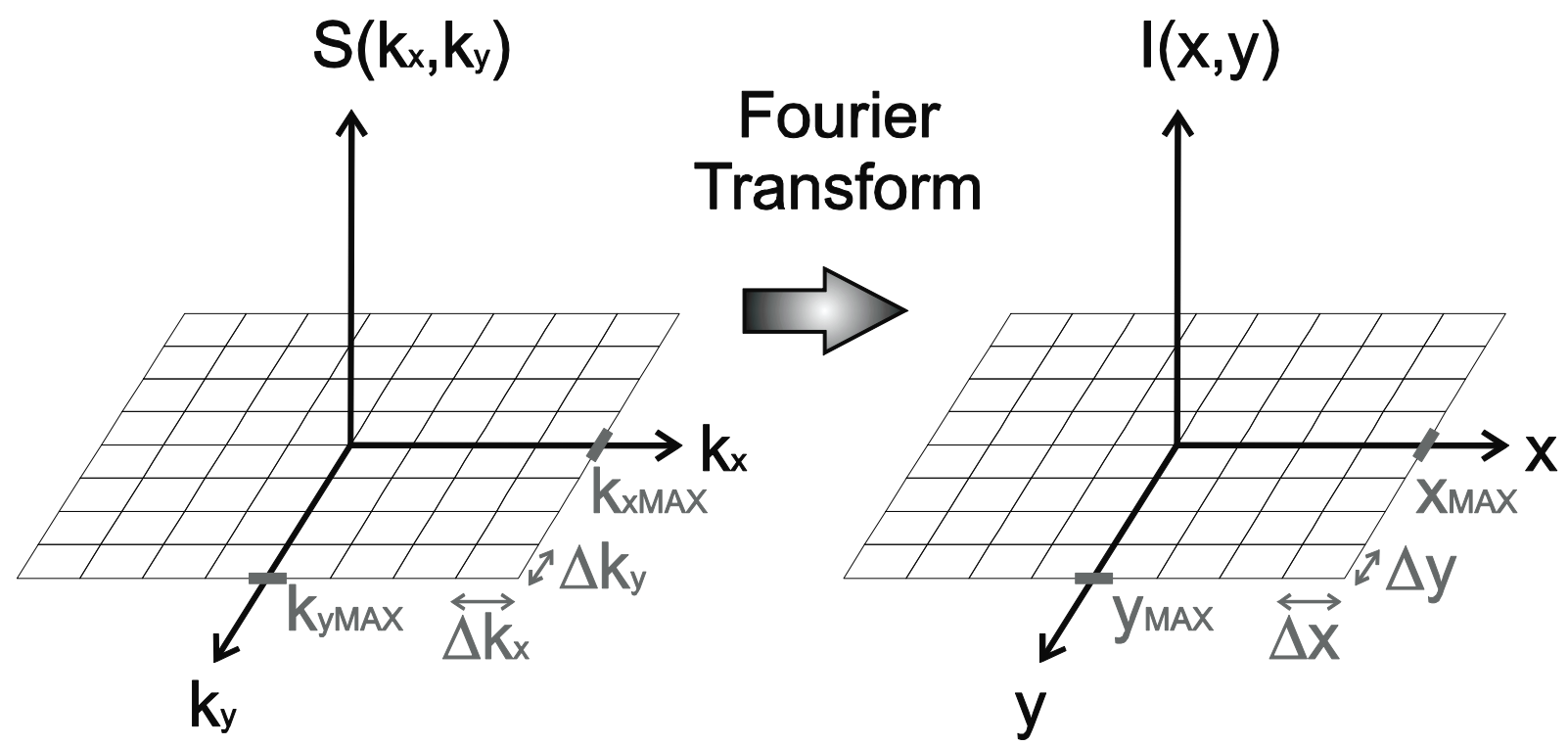

Figure 13: $k$-space and image space. 


\section{Imaging sequences}

To build an MR Image, one needs to sample the k-space. All the different MRI sequences are designed to "fill" the $k$-space efficiently and rapidly. Some classical MRI sequences are illustrated in figure 14 . The effect of a $180^{\circ}$ pulse can be represented simply in the k-space: it inverts the position with respect to the center of the $k$-space. So it can be shown that

$$
S\left(k_{x}, k_{y}\right) \stackrel{180^{\circ}}{\Rightarrow} S^{*}\left(k_{x}, k_{y}\right)=\left[\iint_{\text {slide }} I(x, y) e^{-i 2 \pi\left[k_{x} x+k_{y} y\right]} d x d y\right]^{*}=\iint_{\text {slide }} I(x, y) e^{-i 2 \pi\left[-k_{x} x+-k_{y} y\right]} d x d y=S\left(-k_{x},-k_{y}\right)
$$

For each sequence, figure 14 illustrates on the right the sequences of radiofrequency pulses and gradients $\left(G_{z}, G_{x}, G_{y}\right)$, and on the left the corresponding $k$-space filling trajectory. Gradient echo (fig 14a) and spin echo gradient (fig 14b) sequences fill a line of $k$-space at each repetition of the sequence. This logical scheme is however very time consuming since between two sequences a delay $T_{\mathrm{R}}$ should be respected, in order to allow a complete relaxation of the proton magnetic moments. This is necessary to ensure a maximum signal in the transverse plane, just after the $90^{\circ}$ pulse. Ideally, $T_{\mathrm{R}} \gtrsim 5 T_{1}$. FLASH (Fast Low Angle SHot) sequence (fig 14c) makes it possible to find a compromise between the acquisition time and the signal-to-noise ratio, by using smaller $T_{\mathrm{R}}$ values $\left(T_{\mathrm{R}}<<5 \mathrm{~T}_{1}\right)$ and adjusting the flip angle to the Ernst angle in order to obtain the optimum signal:

$$
\alpha=\operatorname{arcos}\left(e^{-\frac{T_{R}}{T_{1}}}\right)
$$

In this sequence, a spoil gradient (squared surface on figure 14c) is often applied along the $y$ direction, just after the acquisition, in order to destroy the residual signal before the next flip of the magnetization. Faster $k$-space filling can be obtained by FSE (Fast Spin Echo, fig 14d) and EPI (Echo Planar Imaging, fig 14e) sequences. Multiple lines are then encoded in a single shot, which allows a tremendous time gain. This is achieved by multiple echoes (just like in a truncated CPMG sequence) for different phase-encoding gradients in FSE. Rewinding phase gradients (gray surface in fig 14d) 
have to be applied before each $180^{\circ}$ pulse to allow accurate phase encoding of the next echo. A different strategy is used in EPI imaging: multiple gradient echoes are used after the initial spin echo, obtained thanks to a $180^{\circ}$ pulse. Short pulses of phase-encoding gradients (called "blips") are used to jump from a horizontal line of the $k$-space to another before each new gradient echo. 
(a)
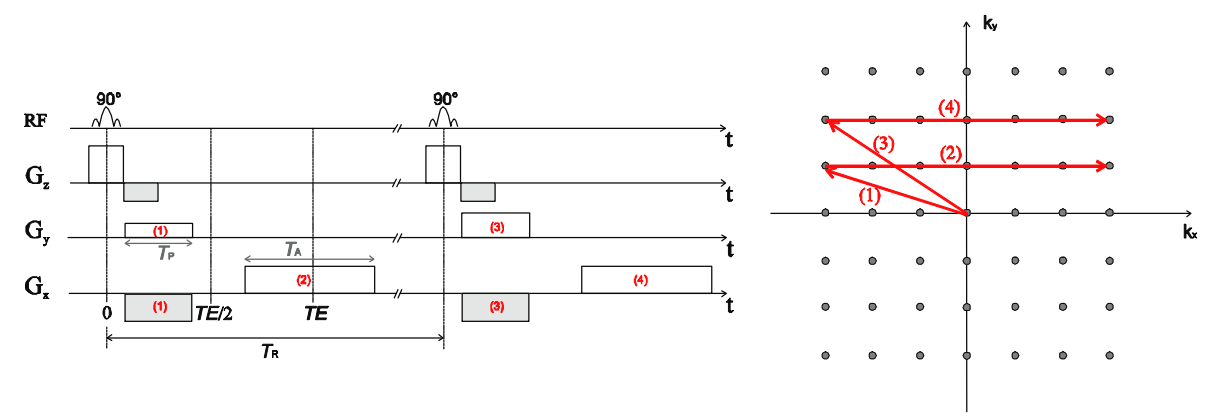

(b)
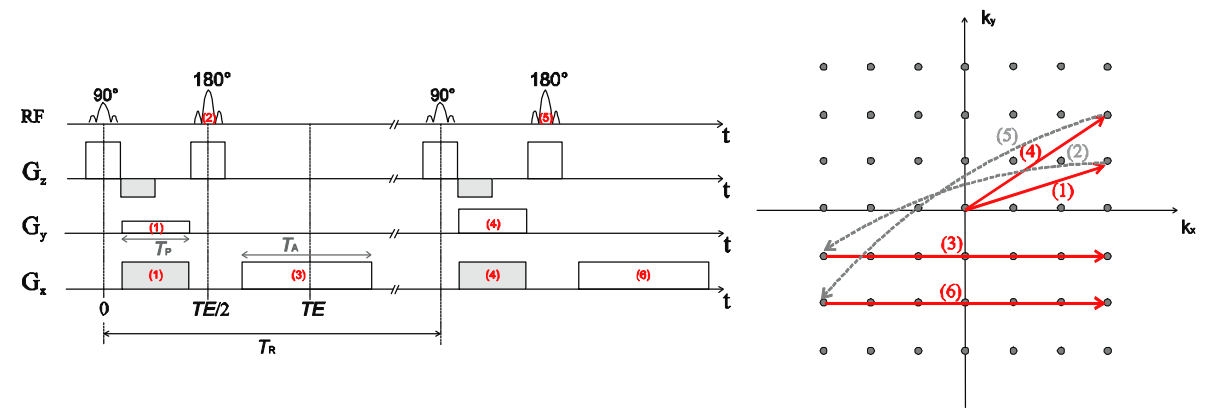

(c)
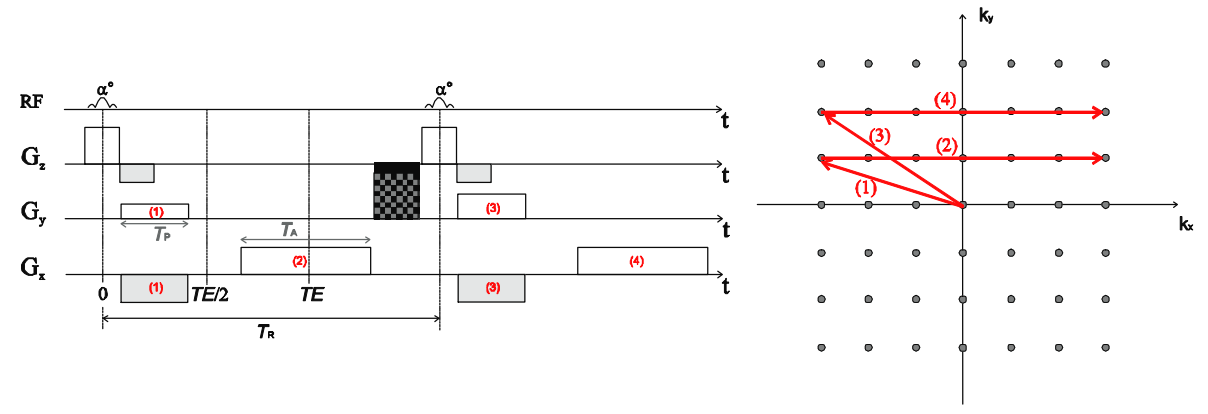

(d)
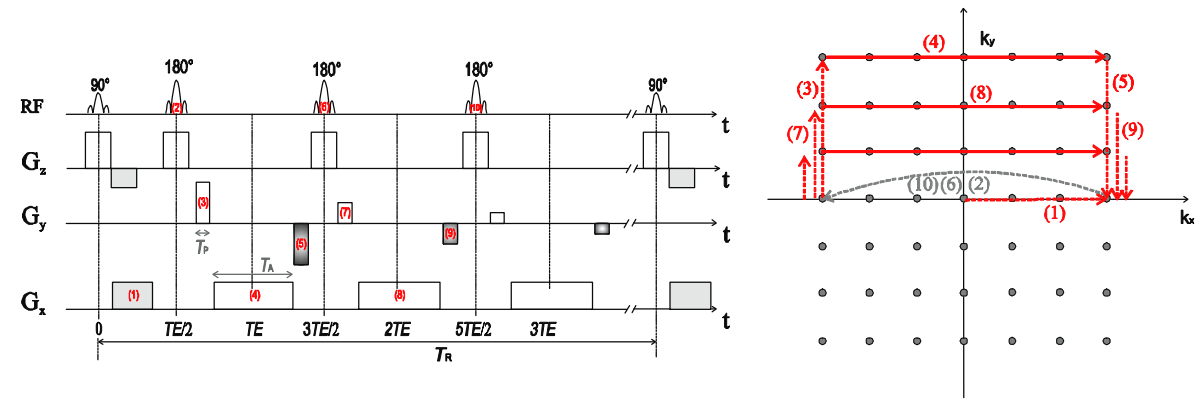

(e)
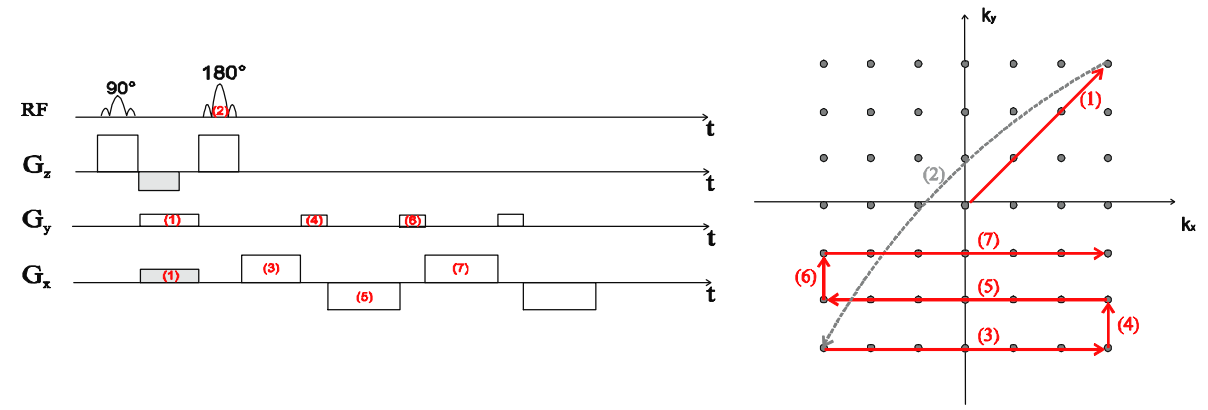

Figure 14: Pulse and gradient succession of some important MRI sequences and the corresponding trajectory in the $k$-space: (a) gradient echo, (b) spin echo, (c) FLASH, (d) FSE and (e) EPI. 


\section{Contrast, $T_{1}$ and $T_{2}$ weighting}

Contrast is by definition the relative difference in intensities of two adjacent pixels (picture elements):

$$
C=\frac{I_{1}-I_{2}}{I_{1}+I_{2}}
$$

Where $I_{1}$ and $I_{2}$ are the signal intensities of pixels 1 and 2 .

Contrast can be caused by differences in proton density $\rho$. This explains the natural contrast observed on MR Images between bones (low $\rho$ ) and fat (high $\rho$ ), for example. Differences in the relaxation times $T_{1}$ and $T_{2}$ can also create contrast. However, the influence of the relaxation times is dependent on the type of MRI sequence and on the sequence parameters. If the sequence is sensitive to $T_{1}\left(T_{2}\right)$, one speaks of $T_{1}$-weighting ( $T_{2}$-weighting). To understand this relaxation-time weighting, let us imagine the simple case of a spin echo sequence (fig 14b). The signal in a pixel is given by:

$$
S(t) \prec \rho_{H}\left(1-e^{-T_{R} / T_{1}}\right) \cdot e^{-T E / T_{2}}
$$

$\rho_{H}$ is the proton density. Two important parameters are the repetition time $T_{R}$ and the echo time TE. If two regions (region A and region B) of the imaged slice (figure 15) present different relaxation times $\left(T_{1 \mathrm{~A}}>T_{1 \mathrm{~B}}\right.$ and $\left.T_{2 \mathrm{~A}}>T_{2 \mathrm{~B}}\right)$, how will $T_{\mathrm{R}}$ and $\mathrm{TE}$ values influence the contrast between $\mathrm{A}$ and $\mathrm{B}$ ?

- If $T_{\mathrm{R}} \sim T_{1 \mathrm{~A}}$ and $T E \ll T_{2 \mathrm{~B}}$, then longitudinal relaxation is not complete between two successive $90^{\circ}$ pulses, which means the signal decreased at the second pulse for the protons whose longitudinal relaxation was not complete (region A). The protons belonging to region $\mathrm{B}$ have almost reached the equilibrium magnetic moment when the second $90^{\circ}$ pulse is applied, inducing a large signal after the pulse. In this case, region $\mathrm{B}$ will thus appear bright and region A will appear dark. The fact that $T E \ll T_{2 \mathrm{~B}}$ ensures that 
the transverse relaxation does not occur between the $90^{\circ}$ pulse and the spin echo, so the signal is not affected by differences in $T_{2}$. The obtained image is purely $T_{1}$-weighted.

- If $T_{\mathrm{R}}>>T_{1 \mathrm{~A}}$ and $T E \sim T_{2 \mathrm{~B}}$ the transverse relaxation provokes a loss of signal between the $90^{\circ}$ pulse and the spin echo. This loss will be significant for the protons having the shortest $T_{2}$ (region $\mathrm{B}$ ) while it will be almost negligible for protons with a long $T_{2}$ (region A). Region B will appear dark because of the signal loss and region A will be bright. The condition $T_{\mathrm{R}} \gg T_{1 \mathrm{~A}}$ ensures that the magnetic moments of regions $\mathrm{A}$ and $\mathrm{B}$ completely return to equilibrium between successive $90^{\circ}$ pulses, in order to avoid $T_{1}$-weighting. The image is in this case purely $T_{2}$ - weighted.

- Mixed $T_{1}$ and $T_{2}$ weighting can be obtained by using $T_{\mathrm{R}} \sim T_{1 \mathrm{~A}}$ and $T E \sim T_{2 \mathrm{~B}}$.

- If $T_{\mathrm{R}}>>T_{1 \mathrm{~A}}$ and $T E<<T_{2 \mathrm{~B}}$ the relaxation rates do not affect contrast. The image is weighted by proton density.

$$
T_{\mathrm{iA}}>T_{\mathrm{iB}}
$$
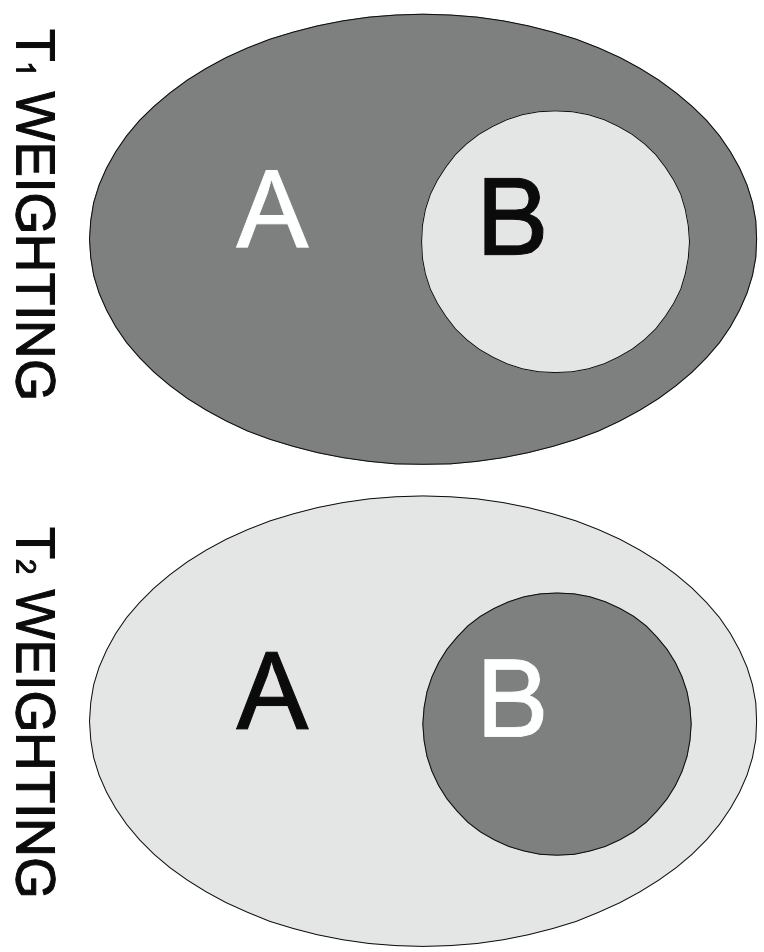

Figure 15: Illustration of $T_{1}$ and $T_{2}$ weighting of an MRI sequence 


\section{Contrast agents ${ }^{14}$}

The natural contrast of MRI is excellent, and in most scans, there is no need to improve it. However, in some important applications, such as tumor detection, exogenous contrast agents (CA) have to be used in order to shorten the relaxation times of some of the tissue protons, which causes an improvement in contrast. In the example of figure 15 , regions $A$ and $B$ could have different relaxation times because of the presence of $C A$ in region $B$ and its absence in region $A$. Two categories of CA are used clinically:

1. Complexes of paramagnetic ions (Gadolinium and Manganese), acting similarly on $T_{1}$ and $T_{2}$. These are composed of a paramagnetic $\mathrm{Gd}^{3+}$ or $\mathrm{Mn}^{2+}$ ion chelated by a molecule with small molecular weight. They are called "positive" contrast agents since their use, in combination with a $T_{1}$-weighted sequence, leads to a stronger signal in their neighborhood ( $\mathrm{B}$ region, bright on the image). The chelation of the ion is necessary because of the toxicity of the free paramagnetic ions. The relaxation induced by paramagnetic ions is mainly caused by the chemical exchange between water molecules coordinated to the paramagnetic complex and bulk water molecules. Paramagnetic contrast agents facilitate the detection of brain tumors. Indeed, these CA are unable to go through the blood-brain barrier, the separation of circulating blood and cerebrospinal blood. However, this barrier is disrupted in the tumor region and the CA can thus accumulate in the tumor. So, tumors, like $B$ region of figure 15 , appear bright in a $T_{1^{-}}$ weighted brain image, as illustrated in image 16. 


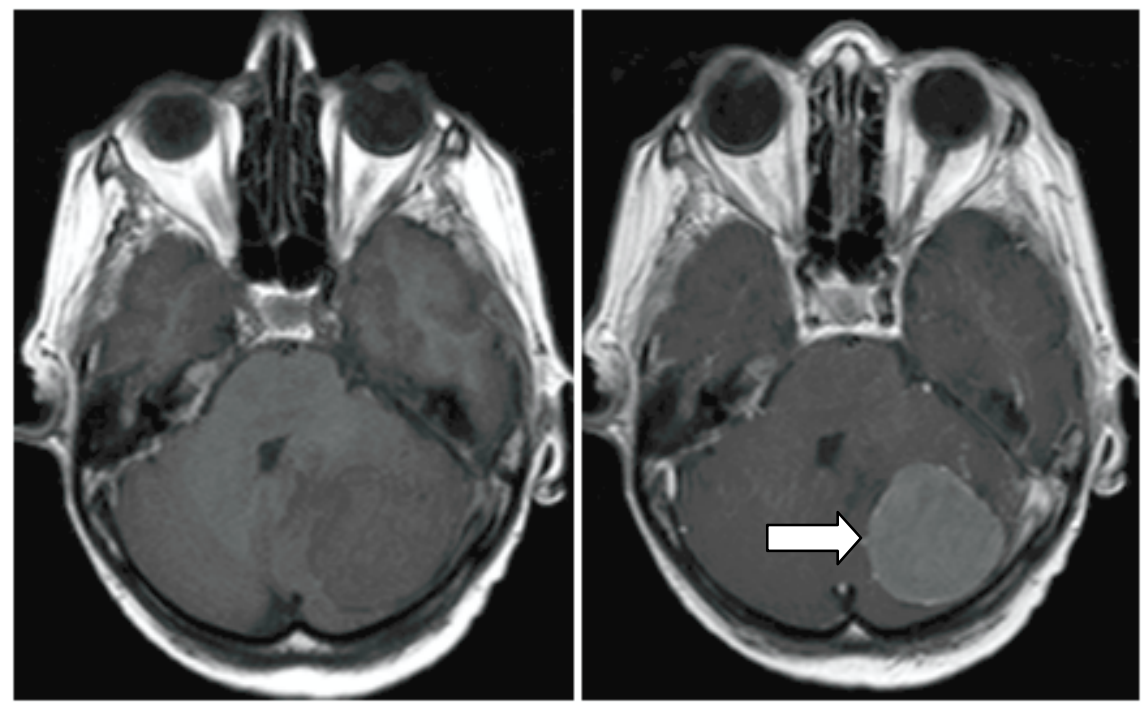

Figure 16: $T_{1}$-weighted spin echo images of the brain, before (left) and after (right) injection of a gadolinium-based contrast agent. The tumor appears brighter on the right image. Images acquired with a Philips Achieva 1.5T MR scanner. Image courtesy of Dr Thierry Metens, ULB-Hôpital Erasme.

2. Superparamagnetic iron oxide nanoparticles, mainly acting on $T_{2}$ and $T_{2}{ }^{*}$. These particles are "negative" contrast agents. Indeed, their use, together with a $T_{2}$-weighted sequence, leads to a lower signal (B region, dark on the image) in their neighborhood. The relaxation mechanism of water protons in the presence of magnetic particles is due to the diffusion of the protons in the magnetic field inhomogeneities created by the particles. Superparamagnetic contrast agents are used to detect liver or spleen tumors. They are absorbed by the reticuloendothelial system but not by tumors. So tumors appear bright, like region A of figure 15, in a "dark" organ on $T_{2}$-weighted images, as illustrated in image 17. 


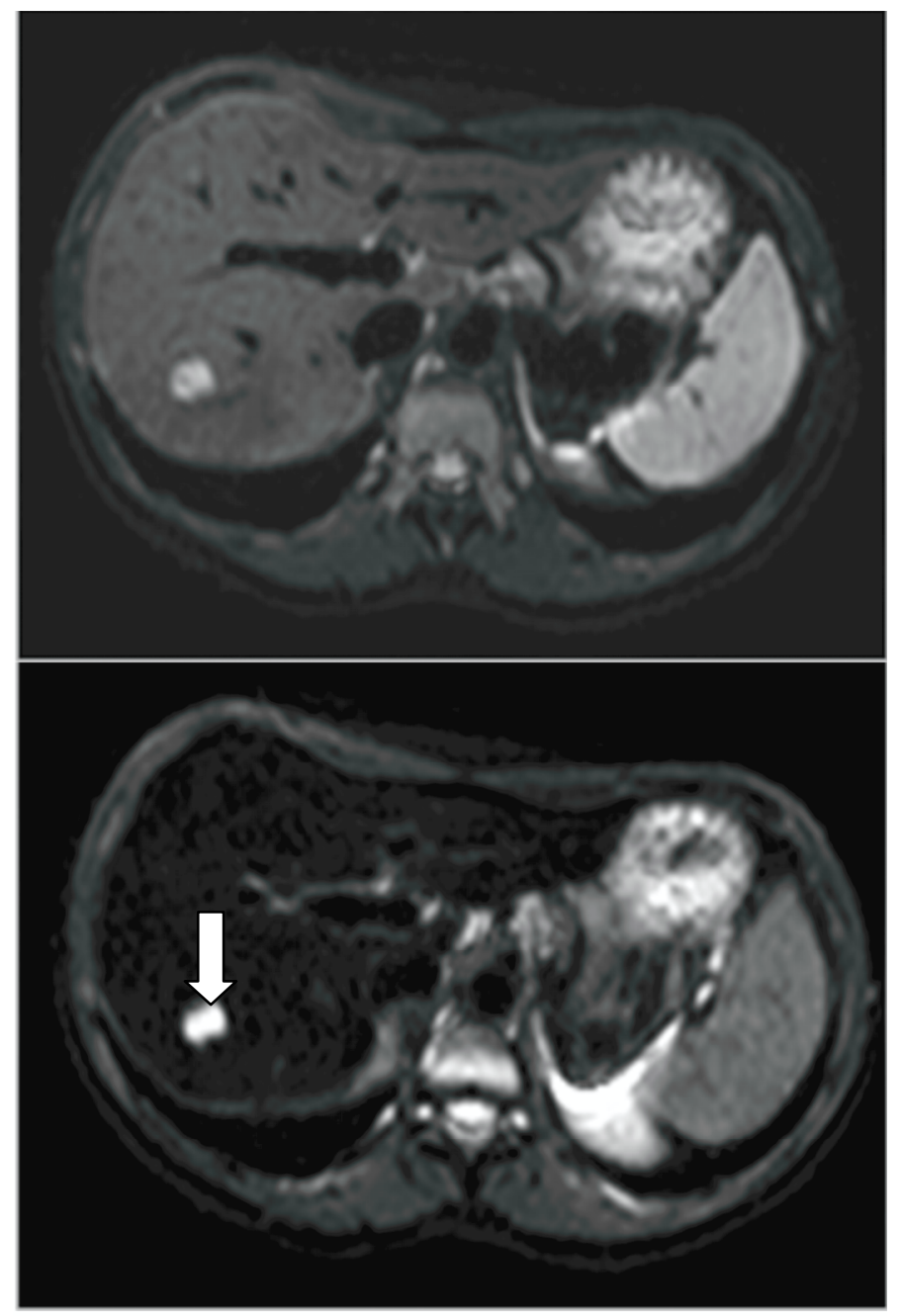

Figure 17: $T_{2}$-weighted spin echo images of the liver, before (above) and after (below) injection of iron oxide nanoparticles. The tumor appears brighter on the second image. Images acquired with a Philips Achieva 1.5T MR scanner. Image courtesy of Dr Thierry Metens, ULB-Hôpital Erasme.

\section{Clinical applications of MRI}

\section{Functional MRI15,16,17,18}

MRI gives information about the form and the structure of organs through anatomic imaging but also about their functions thanks to functional MRI (fMRI). 
The first images of the functioning brain were made in the beginning of the 1990s. fMRI makes it possible to study specific brain functions. The purpose is to locate brain areas involved in different psychomotor and sensorial tasks (view, taste, smell...) or linked to language (listening, reading). An fMRI exam consists of periods of brain activation during which the subject must perform a task (for example, touch his finger, give the name of a pictured object...) alternating with rest periods. The localization of active brain regions is possible thanks to the different magnetic properties of oxyhemoglobin and deoxyhemoglobin. Indeed, hemoglobin, found in red blood cells, can be oxygenated (oxyhemoglobin) and diamagnetic, or non-oxygenated (deoxyhemoglobin) and paramagnetic. The technique is based on the signal modifications due to the variation, in the active brain areas, of the deoxyhemoglobin concentration. Activation of a brain area causes an oxygen consumption increase. So, on the one hand, deoxyhemoglobin concentration increases. On the other hand, increased oxygen consumption induces a large increase in local brain blood flow, rich in oxyhemoglobin. The second effect is much greater: so the ratio of deoxy/oxyhemoglobin decreases. Deoxyhemoglobin being a paramagnetic substance, when its concentration decreases, the $T_{2}{ }^{*}$ weighted signal increases slightly in the active areas. This is the principle of BOLD (Blood Oxygen Level Dependent) contrast. Deoxyhemoglobin can thus be considered as an endogenous contrast agent: it is at the origin of contrast between active and surrounding brain areas. The very slight signal increase makes it necessary to use a statistical treatment of data before any interpretation. The functional image is obtained by making a statistical comparison of numerous images acquired during the rest and activation phases. It is then superimposed on an anatomic image acquired during the same exam (figure 18). Ultra-fast imaging sequences sensitive to $T_{2}{ }^{*}$, like EPI, are often used in fMRI. The temporal resolution is lower than one second and spatial resolution is about one millimeter. 


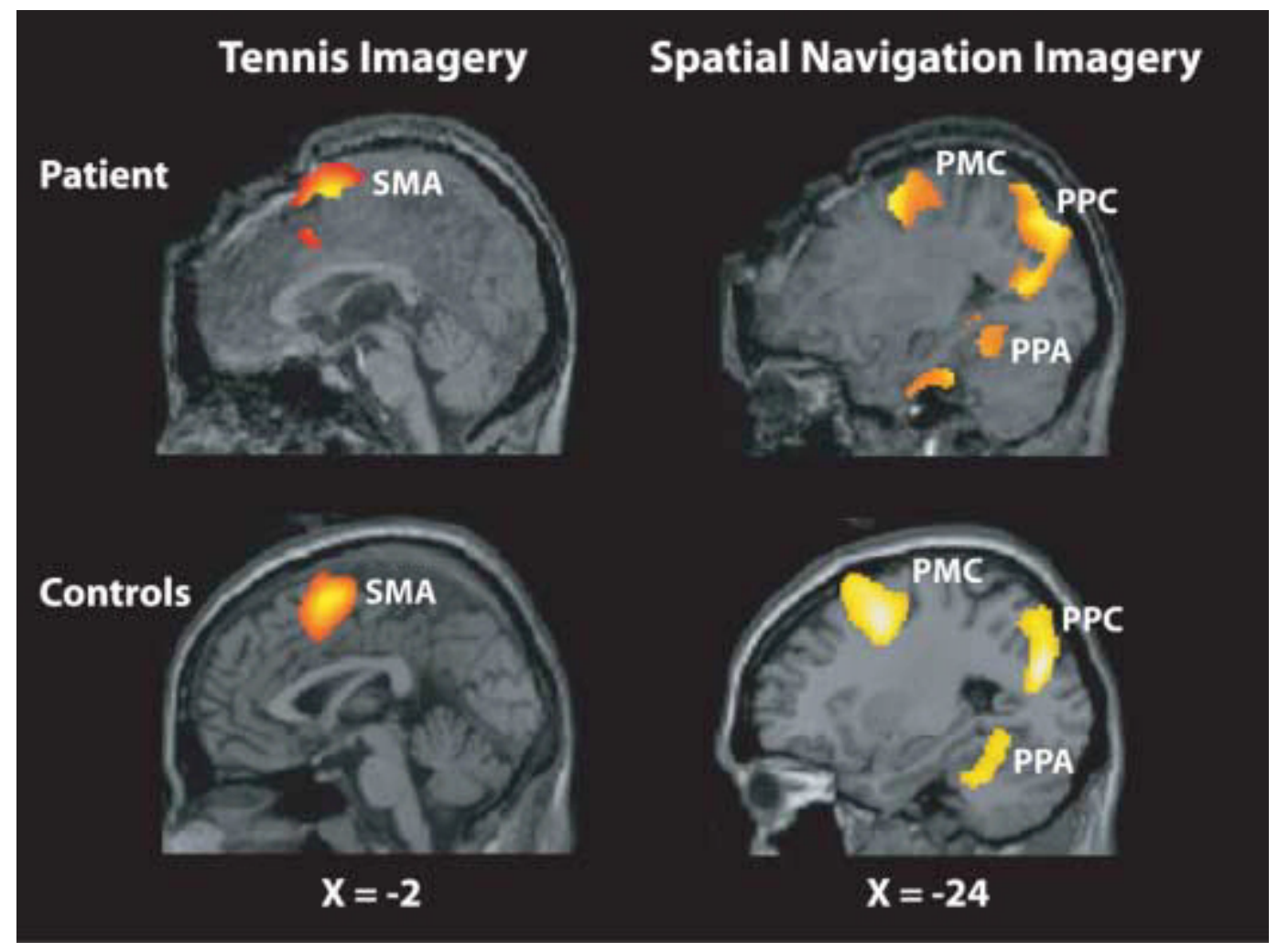

Figure 18: $\mathrm{fMRI}_{\text {study }}{ }^{19}$ showing that a patient in a vegetative state was able to perform two mental imagery tasks. The first task was imagining playing a game of tennis, while the second was imagining visiting all the rooms of her house. The activated brain regions were undistinguishable from those of healthy controls. Reprinted from 12 with permission from AAAS.

Beyond its numerous applications in brain research, $\mathrm{fMRI}$ is also clinically used to locate presurgically functional areas in order to avoid these regions during a tumor resection, for example.

\section{MR Angiography (MRA) $20,21,22,23$}

Vascular MRI techniques are based on flow effects (time-of-flight, phase contrast, fresh blood imaging), or on contrast. These different techniques favor the blood signal over that of the surrounding tissues. Except for fresh blood imaging (FBI), they use $T_{1}$-weighted gradient echo sequences. Parallel imaging can be convenient for all techniques in terms of speed and enhanced image quality. 
Time-f-Flight (TOF) saturates the stationary tissue signal: the magnetic moments of these spins do not have enough time to completely return to equilibrium before the beginning of the imaging sequence. Their signal is weak. On the other hand, TOF favors the inflow effect: the blood flowing into the explored zone is not saturated, so $\mu_{z}$ is maximal. As a consequence, the signal from the flowing blood spins is stronger than that of the saturated stationary spins. The blood vessels thus appear bright on a dark background.

Phase Contrast Angiography (PCA) relies on dephasing of moving spins submitted to a bipolar gradient. After the successive application of two gradients with equal magnitude but opposite directions, stationary spins, on one hand, do not undergo any change in phase, while moving spins (such as in blood), on the other hand, acquire a phase shift because they move constantly through the gradient: they experience a different magnetic field magnitude during the application of the second gradient compared to the first. PCA makes it possible to determine the flow velocity, which is proportional to the dephasing of spins, and also to obtain an MR image of the blood vessels.

Fresh Blood Imaging uses fast or ultra-fast spin echo sequences, synchronized with heartbeats. Arteries are visualized thanks to the longer $T_{2}$ relaxation of blood compared to surrounding tissues.

The contrast-enhanced Magnetic Resonance Angiography (MRA) uses injection of gadolinium chelates to shorten $T_{1}$ and so to increase the blood signal. For this application, the contrast agent must present the longest blood half-life. 


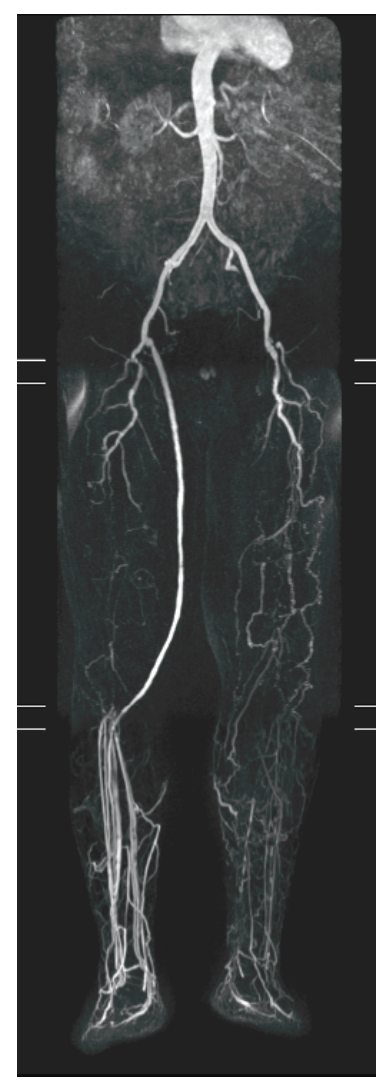

Figure 19: Magnetic resonance angiography. Coronal slice. Images acquired with a Philips Achieva 1.5T MR scanner. Image courtesy of Dr Thierry Metens, ULB-Hôpital Erasme.

MR angiography is used for detection and characterization of vascular walls and blood flow. It allows visualization of the aorta, arteries and veins, mainly in the head, neck, thorax, abdomen, lungs, kidneys and legs, in order to highlight anomalies, such as stenosis, abnormal narrowing, aneurysm, vessel wall dilatation, and risk of rupture.

\section{Diffusion MR Imaging 24,25}

Diffusion MR Imaging makes it possible to study the molecular motions of protons constituting the patient's body. The principle of this technique is to apply strong magnetic field gradients with a spin echo sequence: magnetic moments of static protons are completely "rephased" while moving ones that feel different fields are "dephased", giving different voxel colors in the image. A map of the diffusion tensor can therefore be obtained with such a technique. 
The deduced diffusion coefficient, called apparent diffusion coefficient (ADC), is empirical. The measurement of these quantities provides information allowing, for example, detection of early brain ischemia or observation of brain activation and white matter structure using a non-intrusive method.

Another way to study proton movement is perfusion MRI. In that case, magnetic particles are used as tracers: when they get near a proton, the relaxation rates of the protons are modified. The movement of the magnetic particles can thus be deduced.

\section{Parallel Imaging $26,27,28$}

An interesting way to decrease image acquisition time is to use several coils to detect the MR signal. With such an experimental setup, images are recomposed thanks to the processing of the different coil signals: each one is characterized by the parameters of conventional MRI and by its sensitivity. Thus each coil receives a signal $m_{j}$

$$
m_{j}(\vec{k})=\int I(x, y) s_{j}(x, y) e^{-i \vec{k} \cdot \vec{r}} d \vec{r}
$$

where $s_{j}$ is the sensitivity of the coil $j$. These multiple coils introduce a new degree of freedom that makes it possible to treat the signal more efficiently.

Two main techniques are possible: images can be reconstructed in the image space (SENSE) or in the k-space (SMASH).

- The main idea of the SENSE method is that each coil will produce a partial image of the whole slice. The great difficulty is that one image voxel can be measured by several coils: a correct weighting, based on the coil sensitivities, of the different voxel signals has to be done in order to reconstruct the image. 
- The strategy of the SMASH sequence is to make several ingenious linear combinations of the different coil signals to obtain a more precise interpolated kspace. When this entire space is constructed, the image is then calculated classically using a Fourier Transform.

The main advantages of these methods are that they decrease the acquisition time of an image by limiting the number of repetitious steps of the classical sequences: theoretically, it can be reduced by a factor equal to the number of coils. However, multiple coils increase the complexity of signal processing and decrease the signal-to-noise ratio.

\section{Perspectives and conclusion}

MRI is continuously evolving. New sequences and applications are proposed each week. The technological developments allow to use stronger gradients and higher fields resulting in better resolution and shorter acquisition times.

Fundamental research can lead to useful applications. MRI is used in clinical routine and will be used more intensively in the future. When they discovered the NMR phenomenon in 1946, Bloch and Purcell were probably not imagining the development, thirty years later, of a medical imaging technique based on their results.

\section{Acknowledgments}

The authors thank Robert N Muller for helpful discussions, Dr Thierry Metens (ULB-Hôpital Erasme) and Dr Vincent Denolin (Philips) for allowing them to use MR images to illustrate the article. YG thanks the Bio Imaging lab's team in UA (Antwerp) for micro MRI training in 2007. Ramona Shelby is also acknowledged for her help in preparing the manuscript. 
${ }^{1}$ Abragam A. The principles of nuclear magnetism. Oxford University Press. 1961.

${ }^{2}$ Slichter CP. Principles of magnetic resonance. Springer series in solid state sciences. 1992.

${ }^{3}$ Mc Connell J. The theory of nuclear magnetic relaxation in liquids. Cambridge university press. 1987.

${ }^{4}$ Bloch F. Nuclear induction. Phys Rev 1946;70:460-474.

${ }^{5}$ Bloembergen N, Purcell EM, Pound V. Relaxation effects in nuclear magnetic resonance absorption. Phys Rev 1948;73:679-712.

${ }^{6}$ Hahn EL. Spin echoes. Phys Rev 1950;80:580-594.

${ }^{7}$ Purcell EM, Torrey HC, Pound V. Resonance absorption by nuclear magnetic moments in a solid. Phys Rev 1946;69:37-38.

${ }^{8}$ Liang Z-P, Lauterbur PC. Principles of magnetic resonance imaging. IEEE press series in biomedical engineering. 2000.

${ }^{9}$ Vlaardingerbroek M, den Boer JA. Magnetic resonance imaging. Springer. 2003.

${ }^{10}$ Lauterbur PC. Image formation by induced local interactions: examples employing nuclear magnetic resonance. Nature 1973;242:190-191.

${ }^{11}$ Mansfield P, Grannell PK. NMR 'diffraction' in solids? J Phys C 1973;6:422-426.

${ }^{12}$ Haacke EM, Brown RW, Thompson MR, Venkatesan R. Magnetic Resonance Imaging: physical principles and sequence design. Wiley-Liss (John Wiley \& Sons), New York 1999.

${ }^{13}$ Bittoun J. Theory of k-space: Fourier transformation of a time signal. ESMRMB advanced course, Leuven 2008.

${ }^{14}$ Merbach A, Toth E. The Chemistry of Contrast Agents. Wiley. 2001. 
${ }^{15}$ Ogawa S, Lee TM, Kay AR, Tank DW. Brain magnetic resonance imaging with contrast dependent on blood oxygenation. Proc Natl Acad Sci 1990;87:9868-9872.

${ }^{16}$ Bandettini PA, Wong EC, Hinks RS, Tikofsky RS, Hyde JS. Time course EPI of human brain function during task activation. Magn Reson Med 1992;25:390-397.

${ }^{17}$ Ogawa S, Menon RS, Kim S, Ugurbil K. On the characteristics of functional magnetic resonance imaging of the brain. Annu Rev Biophys Biomol Struct 1998;27:447-474.

${ }^{18}$ Menon RS. Imaging function in the working brain with fMRI. Curr Opin Neurobiol. 2001;11:630-636.

${ }^{19}$ Owen AM, Coleman MR, Boly M, Davis MH, Laureys S, Pickards JD. Detecting awareness in the vegetative state. Science 2006;313:1402.

${ }^{20}$ Edelman RR, Mattle HP, Atkinson DJ, Hoogewoud HM. MR angiography: review article. Am J Roentgenol 1990;154:937-946

${ }^{21}$ Miyasaki M, Roder F. Fresh Blood Imaging: New perspectives in MR angiography. Visions 2007;11:40-45.

${ }^{22}$ Goyen M, Ruehm SG, Debatin JF. MR-angiography: the role of contrast agents. Eur J Radiol 2000;34:247256.

${ }^{23}$ Meaney JFM, Boyle G, O'Keeffe S. Contrast-enhanced magnetic resonance angiography: Current status, theoretical limitations and future potential. Radiography 2007;13:e31-e44.

24 Luypaert R, Boujraf S, Sourbron S, Osteaux M. Diffusion and perfusion MRI: basic physics. Neurotherapeutics 2007;4:499-510.

${ }^{25}$ Le Bihan L. Looking into the functional architecture of the brain with diffusion MRI. Nat. Rev. Neurosci. 2003;4:469-480.

${ }^{26}$ Katscher U, Börnert P. Parallel Magnetic Resonance Imaging. Neurotherapeutics 2007;4:499-510. 
${ }^{27}$ Pruessmann KP, Weiger M, Scheidegger MB, Boesiger P. SENSE: sensitivity encoding for fast MRI. Magn Reson Med 2002;47:1202-1210.

${ }^{28}$ Sodickson DK., Manning WJ. Simultaneous acquisition of spatial harmonics (SMASH): fast imaging with radiofrequency coil arrays. Magn Reson Med 1997;38:591-603 